\title{
State Space Models and MIDAS Regressions*
}

\author{
Jennie Bai ${ }^{\dagger} \quad$ Eric Ghysels ${ }^{\ddagger} \quad$ Jonathan H. Wright $^{\S}$
}

First Draft: May 2009

This Draft: January 4, 2010

\begin{abstract}
We examine the relationship between MIDAS regressions and Kalman filter state space models applied to mixed frequency data. In general, the latter involves a system of equations, whereas in contrast MIDAS regressions involve a (reduced form) single equation. As a consequence, MIDAS regressions might be less efficient, but also less prone to specification errors. First we examine how MIDAS regressions and Kalman filters match up under ideal circumstances, that is in population, and in cases where all the stochastic processes - low and high frequency - are correctly specified by a linear state space model. We characterize cases where the MIDAS regression exactly replicates the steady state Kalman filter weights. In cases where the MIDAS regression is only an approximation, we compute the approximation error and find it to be small (using two different metrics). We also study how MIDAS regressions perform in comparison to the Kalman filter when the latter is subject to specification errors. Our findings favor MIDAS regressions, as their approximation errors are typically small in comparison to the model specification errors of the Kalman filter. The paper concludes with an empirical application comparing MIDAS and Kalman filtering to predict future GDP growth, using monthly macroeconomic series.
\end{abstract}

${ }^{*}$ The second author benefited from funding by the Federal Reserve Bank of New York through the Resident Scholar Program.

${ }^{\dagger}$ Economist, Capital Markets Function, Federal Reserve Bank of New York, 33 Liberty Street New York, NY 10045, e-mail: jennie.bai@ny.frb.org.

${ }^{\ddagger}$ Department of Finance - Kenan-Flagler Business School and Department of Economics, University of North Carolina, McColl Building, Chapel Hill, NC 27599. e-mail: eghysels@unc.edu.

${ }^{\S}$ Department of Economics, Mergenthaler Hall 457, Johns Hopkins University, 3400 N. Charles Street Baltimore, MD 21218, e-mail: wrightj@jhu.edu. 


\section{Introduction}

Not all economic data are sampled at the same frequency. Financial data are readily available on a (intra-)daily basis, whereas most macroeconomic data are sampled weekly, monthly, quarterly or even annually. The mismatch of sampling frequency has been addressed in the context of state space models by Harvey and Pierse (1984), Harvey (1989), Bernanke, Gertler, and Watson (1997), Zadrozny (1990), Mariano and Murasawa (2003), Mittnik and Zadrozny (2004), Aruoba, Diebold, and Scotti (2009), Ghysels and Wright (2009), Kuzin, Marcellino, and Schumacher (2009), among others.

State space models consist of a system of two equations, a measurement equation which links observed series to a latent state process, and a state equation which describes the state process dynamics. The setup treats the low-frequency data as "missing data" and the Kalman filter is a convenient computational device to extract the missing data. The approach has many benefits, but also some drawbacks. State space models can be quite involved, as one must explicitly specify a linear dynamic model for all the series involved : high-frequency data series, latent high-frequency series treated as missing and the lowfrequency observed processes. The system of equations therefore typically requires a lot of parameters, for the measurement equation, the state dynamics and their error processes. The steady state Kalman gain, however, yields a linear projection rule to (1) extract the current latent state, and (2) predict future observations as well as states.

An alternative approach to dealing with data sampled at different frequencies has emerged in recent work by Ghysels, Santa-Clara, and Valkanov (2002), Ghysels, Santa-Clara, and Valkanov (2006) and Andreou, Ghysels, and Kourtellos (2008a) using so called MIDAS, meaning Mi(xed) Da(ta) S(ampling), regressions. ${ }^{1}$ Recent work has used the regressions in the context of improving quarterly macro forecasts with monthly data (see e.g. Armesto, Hernandez-Murillo, Owyang, and Piger (2008), Clements and Galvão (2008a), Clements and Galvão (2008b), Galvão (2006), Schumacher and Breitung (2008), Tay (2007)), or improving quarterly and monthly macroeconomic predictions with daily financial data (see e.g. Andreou, Ghysels, and Kourtellos (2008b), Ghysels and Wright (2009), Hamilton (2006), Tay (2006)).

\footnotetext{
${ }^{1}$ The original work on MIDAS focused on volatility predictions, see e.g. Alper, Fendoglu, and Saltoglu (2008), Chen and Ghysels (2009), Engle, Ghysels, and Sohn (2008), Forsberg and Ghysels (2006), Ghysels, Santa-Clara, and Valkanov (2005), Ghysels, Santa-Clara, and Valkanov (2006), León, Nave, and Rubio (2007), among others.
} 
The purpose of this paper is to examine the relationship between MIDAS regressions and the linear filter that emerges from a steady state Kalman filter. The theory of the Kalman filter applies, strictly speaking, to linear homoskedastic Gaussian systems and yields an optimal filter in population. Consequently, in population, MIDAS regressions can at best match the optimal filter. However, there are two important limitations to this result. First, it applies only in population, ignoring parameter estimation error. Second, it of course assumes that the state space model is correctly specified-state space model predictions can be suboptimal if the regression dynamics are mis-specified. MIDAS regressions provide linear projections given the (high- and low-frequency) regressors without specifying their data generating process. Hence, MIDAS regressions are less prone to mis-specification. This is particularly relevant for high-frequency financial data which feature conditional heteroskedasticity and therefore do not fit within the standard homoskedastic Gaussian state space format. Thus, either because of greater robustness to mis-specification,or because of parsimony, the MIDAS model may end up doing better than the state space model in practice.

The first objective of this paper is to examine how MIDAS regressions and Kalman filters match up under ideal circumstances, that is in population, and in cases where all the stochastic processes - low and high frequency - are correctly specified by a linear state space model.

One important contribution of the paper is that we show the exact relationship between the steady state Kalman filter and various MIDAS regressions. By exact relationship we mean that a MIDAS regression can be viewed as a reduced form expression for the linear projection that emerges from the steady state Kalman filter. In the case of mixed sampling frequencies this steady state Kalman filter has a periodic structure and this maps exactly into a multiplicative MIDAS regression model considered by Chen and Ghysels (2009) and Andreou, Ghysels, and Kourtellos (2008b). This multiplicative MIDAS regression consists of a parameter-driven aggregation of the high-frequency data, combined with the low-frequency observations using a ADL or autoregressive distributed lag model. We show that the multiplicative scheme exactly matches the periodic features of the steady state Kalman gain that drives the state space model filter.

Next, we examine the cases where the MIDAS regression is only an approximation. For those cases, we compute the approximation error, either in terms of forecast mean square errors or in terms of differences in weights, and we find that the approximation errors, regardless of the metric chosen, are very small. 
The Kalman filter is more prone to specification errors, as noted before. Therefore we also examine how MIDAS regressions perform in comparison to the Kalman filter when the latter is mis-specified. Our findings favor MIDAS regressions, as their approximation errors are typically small in comparison to the model specification errors of the Kalman filter.

Finally, the paper concludes with an empirical study similar to that of Kuzin, Marcellino, and Schumacher (2009). Our empirical studies differ in many important ways. First, Kuzin, Marcellino, and Schumacher (2009) adopt the so called mixed frequency VAR framework of Zadrozny (1990) whereas we adopt the approach of Nunes (2005). The latter has at least two

advantages, (1) it handles nowcasting - predicting during the course of quarter as monthly or daily data become available - well and (2) it is built on the factor approach of Stock and Watson (1989), Forni, Hallin, Lippi, and Reichlin (2000), Stock and Watson (2002), among others, widely used in the recent macro forecasting literature. We find the discrepancies between MIDAS and Kalman filtering implementations to often be small - although in some cases the Kalman filter can perform less well than MIDAS regressions - perhaps testimony of specification error issues.

The paper is organized as follows. In section 2, we introduce the state space model of Nunes (2005) and derive its relationship with MIDAS regressions. In this section we characterize cases where the MIDAS regression is an exact reduced form representation of the steady state Kalman filter. Section 3 computes measures of the discrepancy between the Kalman filter and MIDAS regressions in cases where the state space model is correctly specified and the MIDAS regression is only an approximation to the Kalman filter, and also considers cases in which the Kalman filter is mis-specified. Section 4 contains the empirical work, and section 5 concludes.

\section{State space models and MIDAS regressions}

We consider a dynamic factor model:

$$
F_{t+j / m}=\sum_{l=1}^{p} \Phi_{l} F_{t+(j-l) / m}+\eta_{t+j / m} \quad \forall t=1, \ldots, T, \quad j=0, \ldots, m-1
$$

where $F_{t}$ is a $n_{f} \times 1$ dimensional vector process and the matrices $\Phi_{l}$ are $n_{f} \times n_{f}$, with $\eta$ being an i.i.d. zero mean Gaussian error process with diagonal covariance matrix $\Sigma_{\eta}=$ 
$\operatorname{diag}\left(\sigma_{i, \eta}^{2}, i=1, \ldots, n_{f}\right)$. Besides the time scale, the above equation is a typical multi-factor model used for instance by Stock and Watson (1989), Forni, Hallin, Lippi, and Reichlin (2000), Stock and Watson (2002), Bai and Ng (2004), among others. In anticipation of the mixed frequency sampling scheme, we adopt a time scale expressed in a form that easily accommodates such mixtures. For example, with $m=3$ we will have monthly data sampled every quarter, or with $m=22$ we will have daily data sampled every month. The monthly/quarterly combination will be most relevant for the empirical application and simulations in later sections, but for the purpose of generality we start with a generic setup. We have two types of data: (1) time series sampled at a low frequency - every $t$, and (2) time series sampled at high frequency - every $t+j / m j=0, \ldots, m-1$. We will make two convenient simplifications that depart from generality. First, we assume that there is only one low-frequency process and call it $y_{t}$. It would be easy to generalize this to a vector process. Yet, our focus on single equation MIDAS regressions prompts us to consider a single series - otherwise we would have a system of MIDAS regressions. Moreover, focussing on a single low-frequency series is the most common situation involving macroeconomic forecasting of say quarterly GDP growth, or of inflation, etc., using a collection of higher frequency (monthly/weekly/daily) series. Second, we consider the combination of only two sampling frequencies. For example, we do not consider say the combination of daily, weekly, monthly data or daily, monthly, quarterly, etc. This simplification is made only to avoid more cumbersome notation.

The high-frequency data, denoted $x_{i, t-j / m}$ for $i=2, \ldots, n$, relates to the factors as follows:

$$
x_{i, t+j / m}=\gamma_{i}^{\prime} F_{t+j / m}+u_{i, t+j / m} \quad i=2, \ldots, n \quad \forall t \quad j=0, \ldots, m-1
$$

where $\left\{\gamma_{i}\right\}$ are $n_{f} \times 1$ vectors and:

$$
d_{i}\left(L^{1 / m}\right) u_{i, t+j / m}=\varepsilon_{i, t+j / m} \quad d_{i}\left(L^{1 / m}\right) \equiv 1-d_{1 i} L^{1 / m}-\ldots-d_{k i} L^{k / m} \quad \forall i
$$

where the lag operator $L^{1 / m}$ applies to high-frequency data, i.e. $L^{1 / m} u_{i, t} \equiv u_{i, t-1 / m}$, and the $\varepsilon$ s are i.i.d. normal with mean zero and variance $\sigma_{\varepsilon}^{2}$ and are mutually independent. If the low-frequency process were observed at high frequency, it would similarly relate to the factors as follows:

$$
y_{t+j / m}^{*}=\gamma_{1}^{\prime} F_{t+j / m}+u_{1, t+j / m} \quad \forall t \quad j=0, \ldots, m-1
$$


with $u_{1, t+j / m}$ having an $\mathrm{AR}(\mathrm{k})$ representation as in (2.3), denoting $y^{*}$ as the process which is not directly observed. The observed low-frequency process $y$ relates to the $y^{*}$ via a linear aggregation scheme:

$$
y_{t+j / m}^{c}=\Psi_{j} y_{t+(j-1) / m}^{c}+\theta_{j} y_{t+j / m}^{*}
$$

where $y_{t}$ is equal to $y_{t}^{c}$ for integer $t$, and is not observed otherwise. The above scheme, also used by Harvey (1989) and Nunes (2005), covers both stock and flow aggregation, and $y_{t}^{*}$ is a cumulator variable. We henceforth consider the case of stock variable only (setting $\Psi_{j}=1$ ( $j \neq 0, m, 2 m \ldots)$ and $\theta_{j}=1(j=0, m, 2 m \ldots)$ where $1($.$\left.) denotes the indicator function \right)$. However, if we were instead to pick $\Psi_{j}=1(j \neq 0, m, 2 m \ldots)$ and $\theta_{j}=1 / m \forall j$, then this would correspond to a flow variable.

\subsection{Periodic Data Structure and Steady State Predictions}

The purpose of this subsection is to derive a steady state Kalman filtering formula that will be used in the next subsections for comparisons with MIDAS regressions. The material in this section is general and uses some derivations that appear in Assimakis and Adam (2009). The above equations yield a periodic state space model with measurement equation:

$$
Y_{t}^{j}=Z_{j} \alpha_{t+j / m} \quad\left\{\begin{array}{lr}
Y_{t}^{j}=\left(y_{t}, x_{2, t}, \ldots, x_{n, t}\right)^{\prime} & j=0 \\
Y_{t}^{j}=\left(x_{2, t+j / m}, \ldots, x_{n, t+j / m}\right)^{\prime} & 0<j \leq m-1
\end{array}\right.
$$

where

$$
\begin{gathered}
Z_{0}=\left[\begin{array}{cccc}
\gamma_{1}^{\prime} & & \\
\gamma_{2}^{\prime} & O_{n \times n_{f}(p-1)} & I_{n} & O_{n \times n(k-1)} \\
: & & \\
\gamma_{n}^{\prime} & &
\end{array}\right] \\
Z_{j}=\left[\begin{array}{cccc}
\gamma_{2}^{\prime} & & I_{n-1} & O_{(n-1) \times n(k-1)} \\
: & O_{(n-1) \times n_{f}(p-1)} &
\end{array}\right]
\end{gathered}
$$

for $0<\mathrm{j} \leq \mathrm{m}-1$ and state vector

$$
\alpha_{t+j / m}=\left(F_{t+j / m}^{\prime}, \ldots, F_{t+(j-p+1) / m}^{\prime}, u_{t+j / m}^{\prime}, \ldots, u_{t+(j-k+1) / m}^{\prime}\right)^{\prime}
$$


where $u_{t+j / m}=\left(u_{1, t+j / m}, \ldots, u_{n, t+j / m}\right)^{\prime}$.

The transition equation is:

$$
\alpha_{t+j / m}=F \alpha_{t+(j-1) / m}+R \zeta_{t+j / m}
$$

where

$$
\begin{gathered}
F=\left[\begin{array}{cccc}
\Phi_{1} \ldots \Phi_{p-1} & \Phi_{p} & O_{n_{f} \times(k-1) n} & O_{n_{f} \times n} \\
I_{(p-1) n_{f}} & O_{(p-1) n_{f} \times n_{f}} & O_{(p-1) n_{f} \times(k-1) n} & O_{(p-1) n_{f} \times n} \\
O_{n \times(p-1) n_{f}} & O_{n \times n_{f}} & D_{1} \ldots D_{k-1} & D_{k} \\
O_{(k-1) n \times(p-1) n_{f}} & O_{(k-1) n \times n_{f}} & I_{(k-1) n} & O_{(k-1) n \times n}
\end{array}\right] \\
R=\left[\begin{array}{cc}
I_{n_{f}} & O_{n_{f} \times n} \\
O_{(p-1) n_{f} \times n_{f}} & O_{(p-1) n_{f} \times n} \\
O_{n \times n_{f}} & I_{n} \\
O_{n(k-1) \times n_{f}} & O_{n(k-1) \times n}
\end{array}\right]
\end{gathered}
$$

$D_{i}=\operatorname{diag}\left(d_{l, i}, l=1, \ldots, n\right)$ and $\zeta_{t+j / m}=\left(\eta_{t+j / m}^{\prime}, \varepsilon_{1, t+j / m}, \ldots \varepsilon_{n, t+j / m}\right)^{\prime}$. Let $\Sigma_{\zeta}$ denote the variance-covariance matrix of $\zeta_{t+j / m}$.

The above state space model is periodic as it cycles to the data release pattern that repeats itself every $m$ periods. Such systems have a (periodic) steady state (see e.g. Assimakis and Adam (2009)). If we let $P_{j \mid j-1}$ denote the steady state covariance matrix of $\alpha_{t+j / m \mid t+(j-1) / m}$, then the equations:

$$
\begin{aligned}
P_{j+1 \mid j} & =R \Sigma_{\zeta} R^{\prime}+F P_{j \mid j-1} F^{\prime}-F P_{j \mid j-1} Z_{j}^{\prime}\left[Z_{j} P_{j \mid j-1} Z_{j}^{\prime}\right]^{-1} Z_{j} P_{j \mid j-1} F^{\prime} \quad j=0, \ldots, m-2 \\
P_{0 \mid-1} & =R \Sigma_{\zeta} R^{\prime}+F P_{2 \mid 1} F^{\prime}-F P_{2 \mid 1} Z_{j}^{\prime}\left[Z_{j} P_{2 \mid 1} Z_{j}^{\prime}\right]^{-1} Z_{j} P_{2 \mid 1} F^{\prime} \quad j=m-1
\end{aligned}
$$

must be satisfied and $P_{j \mid j-1}=P_{j+m \mid j+m-1}, \forall j$. The periodic steady state Kalman gain is therefore:

$$
K_{j \mid j-1}=P_{j \mid j-1} Z_{j}^{\prime}\left[Z_{j} P_{j \mid j-1} Z_{j}^{\prime}\right]^{-1}
$$

with $K_{j \mid j-1} \equiv K_{j+m \mid j-1+m}, \forall j$. When we define the extraction of the state vector as:

$$
\hat{\alpha}_{(t+j / m) \mid(t+j / m)}=E\left[\alpha_{t+j / m} \mid\left(Y_{\tau}^{\iota}\right)_{\iota \leq j \bmod (m-1)}^{\tau \leq t}\right]
$$


the filtered states are:

$$
\hat{\alpha}_{(t+j / m) \mid(t+j / m)}=A_{j \mid j-1} \hat{\alpha}_{t+(j-1) / m \mid t+(j-1) / m}+K_{j \mid j-1} Y_{t}^{j}
$$

where $A_{j \mid j-1}=F-K_{j \mid j-1} Z_{j} F$ and $Y_{t}^{m}=Y_{t+1}^{0}$.

Suppose we are interested in predicting at low-frequency intervals only, namely $\hat{\alpha}_{(t+k) \mid t}$, for $k$ integer valued, using all available low and high-frequency data. First we note that:

$$
\hat{\alpha}_{(t+k) \mid(t+k)}=\left[\tilde{A}_{1}^{m}\right]^{k} \hat{\alpha}_{t \mid t}+\sum_{i=1}^{m} \sum_{j=1}^{k}\left[\tilde{A}_{1}^{m}\right]^{k-j} \tilde{A}_{i+1}^{m} K_{i \mid i-1} Y_{t+j-1}^{i}
$$

where

$$
\tilde{A}_{j}^{i}= \begin{cases}A_{i \mid i-1} A_{i-1 \mid i-2} \ldots A_{j \mid j-1} & i \geq j \\ I & i<j\end{cases}
$$

Expression (2.12) can be obtained via straightforward algebra - see Assimakis and Adam (2009). If all eigenvalues of $F$ lie inside the unit circle, then all the eigenvalues of $A_{j \mid j-1}$, $j=1, \ldots, m-1$, and are also inside the unit circle, as are the eigenvalues of the product matrices $\left\{\tilde{A}_{j}^{i}\right\}$ (see again Assimakis and Adam (2009)). This implies that we can rewrite (2.12) as:

$$
\begin{aligned}
\hat{\alpha}_{t \mid t}= & \sum_{j=0}^{\infty} \sum_{i=1}^{m}\left[\tilde{A}_{1}^{m}\right]^{j} \tilde{A}_{i+1}^{m} K_{i \mid i-1} Y_{t-j}^{i}=\sum_{j=0}^{\infty}\left[\tilde{A}_{1}^{m}\right]^{j} K_{m \mid m-1}\left(\begin{array}{c}
y_{t-j} \\
x_{2, t-j} \\
\vdots \\
x_{n, t-j}
\end{array}\right) \\
& +\sum_{j=0}^{\infty} \sum_{i=1}^{m-1}\left[\tilde{A}_{1}^{m}\right]^{j} \tilde{A}_{i+1}^{m} K_{i \mid i-1}\left(\begin{array}{c}
x_{2, t-1-j+i / m} \\
\vdots \\
x_{n, t-1-j+i / m}
\end{array}\right)
\end{aligned}
$$

from which forecasts can easily be constructed as $E_{t}\left[y_{t+h}\right]=Z_{0,1} F^{m h} \hat{\alpha}_{t \mid t}$, where $Z_{0,1}$ denotes the first row of the matrix $Z_{0}$. 


\subsection{Using only High-Frequency Data and the DL-MIDAS Regression Model}

Suppose for the moment that we discard the observations of low-frequency data and only consider projections on high-frequency data. The purpose of this subsection is to show that this yields a linear projection linked to a standard steady state (aperiodic) Kalman gain and that this projection has a reduced form representation that maps into what Andreou, Ghysels, and Kourtellos (2008b) called a DL-MIDAS (or Distributed Lag MIDAS) regression.

Unlike the previous subsection, we will first start with a simple example to illustrate the main finding and then we will cover the general case. In particular, let us consider a single factor AR(1) model, instead of the general case in equation (2.1), namely:

$$
f_{t+j / m}=\rho f_{t+(j-1) / m}+\eta_{t+j / m} \quad \forall t=1, \ldots, T, \quad j=0, \ldots, m-1
$$

where $\eta$ is white noise with variance $\sigma_{\eta}^{2}$ and there is only a single high-frequency series related to the latent factor:

$$
x_{t+j / m}=f_{t+j / m}+u_{2, t+j / m} \quad \forall t \quad j=0, \ldots, m-1
$$

instead of equation (2.2), and we also set the slope coefficient equal to one and assume that $u_{2, t-j / m}$ in the above equation is white noise with variance $\sigma_{x}^{2}$.

While it is still the case that:

$$
y_{t}=f_{t}+u_{1, t} \quad \forall t
$$

with $u_{1, t}$ white noise being with variance $\sigma_{y}^{2}$, we assume in this subsection that this measurement is not taken into account. Still we use the fact that:

$$
E\left[y_{t+h} \mid I_{t}^{H F}\right]=\rho^{m h} \hat{f}_{t \mid t}
$$

where $I_{t}^{H F}$ is the high-frequency data set of past $x$ s available at time $t$ and $\hat{f}_{t \mid t}$ is the filtered estimate of the factor conditional on that information set. Let $\kappa$ be the steady state Kalman gain so that $\hat{f}_{t \mid t}=(\rho-\rho \kappa) \hat{f}_{t-1 / m \mid t-1 / m}+\kappa x_{t}$. This implies that:

$$
E\left[y_{t+h} \mid I_{t}^{H F}\right]=\rho^{m h} \kappa \sum_{j=0}^{\infty}(\rho-\rho \kappa)^{j} x_{t-j / m}
$$


Note that $\kappa$ is a function of all the underlying state space parameters. We have deliberately reduced those parameters to a small number by assuming slopes equal to one and assuming that all measurement noise is uncorrelated. What is left are two variances $\sigma_{\eta}^{2}$ and $\sigma_{x}^{2}$.

The above equation compares directly with a DL-MIDAS regression (again ignoring intercepts):

$$
y_{t+h}=\beta \sum_{j=0}^{\bar{K}} w_{j} x_{t-j / m}+\varepsilon_{t} \quad \forall t
$$

where the weighting scheme adopted in Ghysels, Santa-Clara, and Valkanov (2006) and Andreou, Ghysels, and Kourtellos (2008b), among others, is a two-parameter exponential Almon lag polynomial:

$$
w_{j}\left(\theta_{1}, \theta_{2}\right)=\frac{\exp \left\{\theta_{1} j+\theta_{2} j^{2}\right\}}{\sum_{j=1}^{\bar{K}} \exp \left\{\theta_{1} j+\theta_{2} j^{2}\right\}}
$$

Note that the weights are governed by two parameters and scaled such that they add up to one, hence the presence of a slope parameter $\beta$. In the special case of $\theta_{2}=0$ and $\theta_{1}=$ $\ln (\rho-\rho \kappa)$ (assuming $\rho>\rho \kappa)$, the two weighting schemes are identical.

Note two important issues: (1) the DL-MIDAS regression provides an exact fit for the linear projection emerging from the steady state Kalman filter for sufficiently large lag-length $L$, and (2) this exact fit is accomplished with fewer parameters. Indeed, the DL-MIDAS regression under-identifies the state space model parameters $\rho, \sigma_{\eta}^{2}$ and $\sigma_{x}^{2}$ which determine the steady state Kalman gain. Note another important difference: for the MIDAS regressions we do not write down explicit equations for the dynamics of the (high-frequency) regressor $x$. In the case of a state space model this is required - hence the proliferation of parameters - and also the potential danger of specification errors.

In the general case of the model given by equations (2.1)-(2.5) but where only the highfrequency data are used for forecasting, let $K$ denote the steady state Kalman gain, and let

$$
Z=Z_{j}=\left[\begin{array}{cccc}
\gamma_{2}^{\prime} & & \\
: & O_{(n-1) \times n_{f}(p-1)} & I_{n-1} & O_{(n-1) \times n(k-1)} \\
\gamma_{n}^{\prime} & & &
\end{array}\right]
$$

Then (2.13) reduces to 


$$
\hat{y}_{t+h \mid t}=\rho^{m h} \sum_{j=0}^{\infty}(F-K Z F)^{j} K\left(\begin{array}{c}
x_{2, t-j / m} \\
\vdots \\
x_{n, t-j / m}
\end{array}\right)
$$

which is not exactly a MIDAS regression, but may be well approximated by one - a topic which we will address in section 3 .

\subsection{Using Both Low- and High-Frequency Data and the ADL- MIDAS Regression Model}

We will start again with the simple example appearing in the previous subsection, yet this time we also take into account past low-frequency measurements of $y$. For the sake of simplicity we consider the quarterly/monthly data combination. Hence, we are interested in for instance $E\left[y_{t+h} \mid I_{t}^{M}\right]$, where $I_{t}^{M}$ is the mixed data set of past low (quarterly) and high (monthly) frequency data, instead of the linear projection only involving high-frequency data as in equation (2.18). In the latter case we obtained a standard (aperiodic) steady state equation driving the linear projection. Here, however, we deal with a periodic Kalman filter as in subsection 2.1 applied to the model consisting of equations (2.14), (2.15) and (2.16). Then the periodic Kalman gain matrices are:

$$
K_{1 \mid 0}=\left(\begin{array}{c}
\kappa_{1} \\
* \\
*
\end{array}\right), K_{2 \mid 1}=\left(\begin{array}{c}
\kappa_{2} \\
* \\
*
\end{array}\right) \text { and } K_{3 \mid 2}=\left(\begin{array}{cc}
\kappa_{3,1} & \kappa_{3,2} \\
* & * \\
* & *
\end{array}\right) \text {, }
$$

where "*" denotes some element that does not need to be explicitly named. In addition, let us write $\kappa_{3}=\kappa_{3,1}+\kappa_{3,2}$. The state vector is $\alpha_{t+j / m}=\left(f_{t+j / m}, u_{1, t+j / m}, u_{2, t+j / m}\right)^{\prime}$, and we have

$$
F=\left(\begin{array}{lll}
\rho & 0 & 0 \\
0 & 0 & 0 \\
0 & 0 & 0
\end{array}\right)
$$

and the first rows of the matrices $\tilde{A}_{1}^{m}, \tilde{A}_{2}^{m}$ and $\tilde{A}_{3}^{m}$ are $\left(\left(\rho-\rho \kappa_{1}\right)\left(\rho-\rho \kappa_{2}\right)\left(\rho-\rho \kappa_{3}\right), 0, \ldots 0\right)$, $\left(\left(\rho-\rho \kappa_{2}\right)\left(\rho-\rho \kappa_{3}\right), 0, \ldots 0\right)$ and $\left(\rho-\rho \kappa_{3}, 0, \ldots 0\right)$, respectively. From equation $(2.13)$ it then 
follows that:

$$
E\left[y_{t+h} \mid I_{t}^{M}\right]=\rho^{3 h} f_{t \mid t}=\rho^{3 h} \kappa_{3,1} \sum_{j=0}^{\infty} \vartheta^{j} y_{t-j}+\rho^{3 h} \sum_{j=0}^{\infty} \vartheta^{j} x\left(\theta_{x}\right)_{t-j}
$$

where $\vartheta=\left[\left(\rho-\rho \kappa_{1}\right)\left(\rho-\rho \kappa_{2}\right)\left(\rho-\rho \kappa_{3}\right)\right]$, and

$$
x\left(\theta_{x}\right)_{t} \equiv\left[\kappa_{3,2}+\left(\rho-\rho \kappa_{3}\right) \kappa_{2} L^{1 / 3}+\left(\rho-\rho \kappa_{3}\right)\left(\rho-\rho \kappa_{2}\right) \kappa_{1} L^{2 / 3}\right] x_{t}
$$

which is a parameter-driven low-frequency process composed of high-frequency data aggregated at the quarterly level.

The above equation relates to the multiplicative MIDAS regression models considered by Chen and Ghysels (2009) and Andreou, Ghysels, and Kourtellos (2008b). In particular consider the following ADL-MIDAS regression:

$$
y_{t+h}=\beta_{y} \sum_{j=0}^{K_{y}} w_{j}\left(\theta_{y}\right) y_{t-j}+\beta_{x} \sum_{j=0}^{K_{x}} w_{j}\left(\theta_{x}^{1}\right)^{j} x\left(\theta_{x}^{2}\right)_{t-j}+\varepsilon_{t+1}
$$

where $w_{j}\left(\theta_{y}\right), w_{j}\left(\theta_{x}^{1}\right)$ follow an exponential Almon scheme and

$$
x\left(\theta_{x}^{2}\right)_{t-j} \equiv \sum_{k=0}^{m-1} w_{k}\left(\theta_{x}^{2}\right) L^{k / m} x_{t-k / m}
$$

also follows an exponential Almon scheme. Provided that $\rho>0$, equations (2.21) and (2.22) are a special case of this model with $K_{y}=K_{x}=\infty, w_{j}\left(\theta_{y}\right) \propto \exp (\log (\vartheta) j)$, $w_{j}\left(\theta_{x}^{1}\right) \propto \exp (\log (\vartheta) j)$ and $w_{k}\left(\theta_{x}^{2}\right) \propto \exp \left(\theta_{x, 1}^{2} k+\theta_{x, 2}^{2} k^{2}\right)$ where $\theta_{x, 1}^{2}$ and $\theta_{x, 2}^{2}$ are parameters that solve the equations

$$
\begin{gathered}
\log \left\{\left(\rho-\rho \kappa_{3}\right) \kappa_{2} / \kappa_{3,2}\right\}=\theta_{x, 1}^{2}+\theta_{x, 2}^{2} \\
\log \left\{\left(\rho-\rho \kappa_{3}\right)\left(\rho-\rho \kappa_{2}\right) \kappa_{1} / \kappa_{3,2}\right\}=2 \theta_{x, 1}^{2}+4 \theta_{x, 2}^{2}
\end{gathered}
$$

This constructed low-frequency regressor is estimated jointly with the other (MIDAS) regression parameters. Hence, one can view $x\left(\theta_{x}^{2}\right)_{t-j}$ as the best aggregator that yields the best prediction. This ADL-MIDAS regression involves more parameters than the usual specification involving only one polynomial. The multiplicative specification was originally 
suggested in Chen and Ghysels (2009) to handle seasonal patterns (in their case the intradaily seasonal of volatility patterns). Comparing equations (2.21) and (2.23) again yields an exact mapping, if $\rho>0$.

\section{Approximation and Specification Errors}

From the previous section we know that the mapping between the Kalman filter and MIDAS regressions can be exact. We now analyze cases where the MIDAS regression is instead only an approximation. The purpose of this section is to assess the accuracy of a population approximation to the Kalman filter obtained from a MIDAS regression.

We will focus on two cases where MIDAS regressions do not yield an exact mapping with the Kalman filter. A subsection is devoted to each case. The first is a one-factor state space model with measurement errors that are serially correlated over time. The second is a two-factor state space model. The final subsection covers specification errors.

\subsection{One-Factor State Space Model versus MIDAS}

We start again with the example of a single factor AR(1) model in equation (2.14) appearing in Section 2.2, yet allowing for persistence in the measurement errors. For the quarterlymonthly data combination this yields:

$$
\begin{aligned}
& f_{t+j / m}=\rho f_{t+(j-1) / m}+\eta_{t+j / m} \quad \forall t \quad j=0, \ldots, m-1 \\
& y_{t+j / m}^{*}=\gamma_{1} f_{t+j / m}+u_{1, t+j / m} \quad \forall t \quad j=0, \ldots, m-1 \\
& x_{t+j / m}=\gamma_{2} f_{t+j / m}+u_{2, t+j / m} \quad \forall t \quad j=0, \ldots, m-1
\end{aligned}
$$

where

$$
u_{i, t+j / m}-d_{i} u_{i, t+(j-1) / m}=\epsilon_{i, t+j / m} \quad i=1,2 .
$$

Then the periodic Kalman gain matrices are:

$$
K_{1 \mid 0}=\left(\begin{array}{c}
\kappa_{1}^{1} \\
\kappa_{2}^{1} \\
\kappa_{3}^{1}
\end{array}\right), K_{2 \mid 1}=\left(\begin{array}{c}
\kappa_{1}^{2} \\
\kappa_{2}^{2} \\
\kappa_{3}^{2}
\end{array}\right) \text { and } K_{3 \mid 2}=\left(\begin{array}{cc}
\kappa_{1,1}^{3} & \kappa_{1,2}^{3} \\
\kappa_{2,1}^{3} & \kappa_{2,2}^{3} \\
\kappa_{3,1}^{3} & \kappa_{3,2}^{3}
\end{array}\right) \text {. }
$$


The state vector is $\alpha_{t+j / m}=\left(f_{t+j / m}, u_{1, t+j / m}, u_{2, t+j / m}\right)^{\prime}$ and we have

$$
\begin{gathered}
F=\left(\begin{array}{ccc}
\rho & 0 & 0 \\
0 & d_{1} & 0 \\
0 & 0 & d_{2}
\end{array}\right), \\
Z_{j}=\left(\begin{array}{ccc}
\gamma_{2} & 0 & 1
\end{array}\right) \quad 0<j \leq m-1 \\
Z_{0}=\left(\begin{array}{lll}
\gamma_{1} & 1 & 0 \\
\gamma_{2} & 0 & 1
\end{array}\right) \quad j=0 .
\end{gathered}
$$

Correspondingly, since $A_{j \mid j-1}=F-K_{j \mid j-1} Z_{j} F$, we can compute $A_{1 \mid 0}, A_{2 \mid 1}$ and $A_{3 \mid 2}$ appearing respectively in equations (A.1) through (A.3) in Appendix A. Using these matrices we can compute the Kalman filter equation for $h$-quarter-ahead prediction, a long expression appearing in equation (A.4) also in Appendix A. To simplify notation, write the Kalman filter prediction as:

$$
E_{K F}\left(y_{t+h} \mid I_{t}^{M}\right)=\sum_{j=0}^{\infty} w_{y, j}^{K F} y_{t-j}+\sum_{j=0}^{\infty} w_{x, j}^{K F} x_{t-j / m}
$$

and the corresponding MIDAS regression as:

$$
E_{M d s}\left(y_{t+h} \mid I_{t}^{M}\right)=\sum_{j=0}^{\bar{K}} w_{y, j}^{M d s} y_{t-j}+\sum_{j=0}^{3 \bar{K}} w_{x, j}^{M d s} x_{t-j / m}
$$

We will consider two types of MIDAS regression specifications, both relate to the above regression as follows: a multiplicative scheme referring to the ADL-MIDAS regression appearing in equation (2.23), and a 'regular' MIDAS scheme which does not involve the aggregator scheme, but instead has a single polynomial specification for the high-frequency data, namely:

$$
y_{t+h}=\beta_{y} \sum_{j=0}^{K_{y}} w_{j}\left(\theta_{y}\right) y_{t-j}+\beta_{x} \sum_{j=0}^{K_{x}} w_{j}\left(\theta_{x}\right)^{j} x_{t-j / m}+\varepsilon_{t+h}
$$

We will compare the models using two criteria. The first is the prediction error minimization. Assuming that the Kalman Filter weights are negligible beyond lag length $\bar{K}$, let $\Sigma_{x y}$ denote the variance-covariance matrix of $\left(x_{t}, y_{t}^{*}, x_{t-1 / m}, y_{t-1 / m}^{*}, \ldots, x_{t-\bar{K}}, y_{t-\bar{K}}^{*}\right)^{\prime}$, the elements 
of which are as follows:

$$
\begin{gathered}
\operatorname{Cov}\left(y_{t-i / m}^{*}, y_{t-j / m}^{*}\right)=\gamma_{1}^{2} \frac{\rho^{|i-j|} \sigma_{\eta}^{2}}{1-\rho^{2}}+\frac{d_{1}^{|i-j|} \sigma_{y}^{2}}{1-d_{1}^{2}} \\
\operatorname{Cov}\left(x_{t-i / m}, x_{t-j / m}\right)=\gamma_{2}^{2} \frac{\rho^{|i-j|} \sigma_{\eta}^{2}}{1-\rho^{2}}+\frac{d_{2}^{|i-j|} \sigma_{x}^{2}}{1-d_{2}^{2}} \\
\operatorname{Cov}\left(x_{t-i / m}, y_{t-j / m}^{*}\right)=\gamma_{1} \gamma_{2} \frac{\rho^{|i-j|} \sigma_{\eta}^{2}}{1-\rho^{2}}
\end{gathered}
$$

for $i, j=0,1,2, \ldots, 3 \bar{K}$, where $\sigma_{\eta}^{2}=\operatorname{Var}\left(\eta_{t}\right), \sigma_{y}^{2}=\operatorname{Var}\left(\varepsilon_{1, t}\right)$ and $\sigma_{x}^{2}=\operatorname{Var}\left(\varepsilon_{2, t}\right)$. Then, the $h$-quarter-ahead Kalman Filter prediction error is $w_{K F}^{\prime} \Sigma_{x y} w_{K F}$ where the weights appear in Appendix A. Similarly, the corresponding MIDAS prediction error is $w_{M d s}^{\prime} \Sigma_{x y} w_{M d s}$, again with details in the aforementioned Appendix.

We choose the MIDAS parameters to minimize the difference of prediction errors between MIDAS and state space models, that is:

$$
\min \left(w_{M d s}^{\prime} \Sigma_{x y} w_{M d s}-w_{K F}^{\prime} \Sigma_{x y} w_{K F}\right)^{2}
$$

It will be convenient to report the results in relative terms, namely the ratio of prediction error variances:

$$
\frac{P E-M i d a s}{P E-S S}=\frac{w_{M d s}^{\prime} \Sigma_{x y} w_{M d s}}{w_{K F}^{\prime} \Sigma_{x y} w_{K F}} .
$$

An alternative measure that we also consider is an $L^{2}$ distance between the weights:

$$
\mathbb{L}^{2} \equiv \sum_{j=0}^{\bar{K}}\left(w_{x, j}^{K F}-w_{x, j}^{M d s}\right)^{2}+\sum_{j=0}^{\bar{K}}\left(w_{y, j}^{K F}-w_{y, j}^{M d s}\right)^{2}
$$

This comparison will tell us that while the two specifications may be close in terms of prediction error, they may still differ in terms of polynomial weights.

Panel A of Table 1 shows the minimized values of $\mathbb{L}^{2}$ comparing Kalman Filter and MIDAS regressions (regular and multiplicative), with $d=d_{1}=d_{2}, \gamma_{1}=\gamma_{2}=1$ and $\sigma_{\eta}^{2}=\sigma_{y}^{2}=\sigma_{x}^{2}$ $=1$. Results are shown for combinations of $d$ and $\rho$, and the forecast horizons $h=2$ and 4. In Panel $\mathrm{B}$ we also report the values of $\mathbb{L}^{2}$ that correspond to the minimized prediction errors. We do not actually report the results for the prediction error ratios as they are easy 
to summarize - for all combinations of $d$ and $\rho$ the predictions are for all practical purposes equal, i.e. the value of the PE-ratio is numerically extremely close to one uniformly in the parameter space.

For $d=0$ and $\rho>0$, by construction, the multiplicative MIDAS provides a perfect fit to the Kalman Filter, and so both distance measures are equal to zero. In contrast to the multiplicative MIDAS, we do not expect the fit with the regular specification to be exact. Yet the results in Table 1 - Panels A and B - show that the difference between the regular MIDAS and Kalman filter weights is also negligible. For other combinations of $d$ and $\rho$ we occasionally observe some significant differences. However, they are concentrated around the extreme values for either $d(-0.9$ or 0.99$)$ or $\rho$ (also -0.9 or 0.99$)$. The regular MIDAS appears to handle the case $\rho=-0.9$ combined with positively autocorrelated measurement noise better. Conversely, the multiplicative MIDAS better handles the $\rho=0.9$ cases. For all other entries to Table 1 the differences between MIDAS weights and the Kalman filter ones are small with both criteria. The multiplicative MIDAS specification generally yields smaller errors than regular MIDAS. This is somewhat expected since the former provides an exact match for some parameter combinations. It is also worth noting that the impact of forecast horizon appears to be small, judging by the differences between $h=2$ and 4 in both panels of Table 1 .

\subsection{Two-Factor State Space Model versus MIDAS}

The second case we consider where the MIDAS regression is only an approximation is a two-factor state space model:

$$
\begin{aligned}
& F_{t+j / m}=\left(\begin{array}{l}
f_{1, t+j / m} \\
f_{2, t+j / m}
\end{array}\right)=\left(\begin{array}{cc}
\rho_{1} & 0 \\
0 & \rho_{2}
\end{array}\right)\left(\begin{array}{l}
f_{1, t+(j-1) / m} \\
f_{2, t+(j-1) / m}
\end{array}\right)+\left(\begin{array}{c}
\eta_{1, t+j / m} \\
\eta_{2, t+j / m}
\end{array}\right) \quad j=0, \ldots, m-1 \\
& y_{t+j / m}^{*}=\gamma_{1}^{\prime} F_{t+j / m}+u_{1, t+j / m} \quad \forall t \quad j=0, \ldots, m-1 \\
& x_{2, t+j / m}=\gamma_{2}^{\prime} F_{t+j / m}+u_{2, t+j / m} \quad \forall t \quad j=0, \ldots, m-1
\end{aligned}
$$

where

$$
u_{i, t+j / m}-d_{i} u_{i, t+(j-1) / m}=\epsilon_{i, t+j / m} \quad i=1,2 .
$$


Then the periodic Kalman gain matrices are:

$$
K_{1 \mid 0}=\left(\begin{array}{c}
\kappa_{1}^{1} \\
\kappa_{2}^{1} \\
\kappa_{3}^{1} \\
\kappa_{4}^{1}
\end{array}\right), K_{2 \mid 1}=\left(\begin{array}{c}
\kappa_{1}^{2} \\
\kappa_{2}^{2} \\
\kappa_{3}^{2} \\
\kappa_{4}^{2}
\end{array}\right) \text { and } K_{3 \mid 1}=\left(\begin{array}{cc}
\kappa_{1,1}^{3} & \kappa_{1,2}^{3} \\
\kappa_{2,1}^{3} & \kappa_{2,2}^{3} \\
\kappa_{3,1}^{3} & \kappa_{3,2}^{3} \\
\kappa_{4,1}^{3} & \kappa_{4,2}^{3}
\end{array}\right) \text {, }
$$

The state vector is $\alpha_{t+j / m}=\left(f_{1, t+j / m}, f_{2, t+j / m}, u_{1, t+j / m}, u_{2, t+j / m}\right)^{\prime}$ and we have

$$
\begin{gathered}
F=\left(\begin{array}{cccc}
\rho_{1} & 0 & 0 & 0 \\
0 & \rho_{2} & 0 & 0 \\
0 & 0 & d_{1} & 0 \\
0 & 0 & 0 & d_{2}
\end{array}\right), \\
Z_{j}=\left(\begin{array}{cccc}
\gamma_{2,1} & \gamma_{2,2} & 0 & 1
\end{array}\right) 0<j \leq m-1 \\
Z_{0}=\left(\begin{array}{cccc}
\gamma_{1,1} & \gamma_{1,2} & 1 & 0 \\
\gamma_{2,1} & \gamma_{2,2} & 0 & 1
\end{array}\right) j=0 .
\end{gathered}
$$

Correspondingly, since $A_{j \mid j-1}=F-K_{j \mid j-1} Z_{j} F$, we can compute again $A_{1 \mid 0}, A_{2 \mid 1}$ and $A_{3 \mid 2}$ appearing respectively in equations (B.1) through (B.3) in Appendix B.

$$
\begin{aligned}
E\left(y_{t+h} \mid I_{t}^{M}\right) & =E\left(\gamma_{1,1} f_{1, t+h}+\gamma_{1,2} f_{2, t+h}+u_{1, t+h} \mid I_{t}^{M}\right) \\
& =\gamma_{1,1} \rho_{1}^{3 h} E\left(f_{1, t} \mid I_{t}^{M}\right)+\gamma_{1,2} \rho_{2}^{3 h} E\left(f_{2, t} \mid I_{t}^{M}\right)+d_{1}^{3 h} E\left(u_{1, t} \mid I_{t}^{M}\right),
\end{aligned}
$$

we have:

$$
E\left(y_{t+h} \mid I_{t}\right)=\left(\begin{array}{llll}
\gamma_{1,1} \rho_{1}^{3 h} & \gamma_{1,2} \rho_{2}^{3 h} & d_{1}^{3 h} & 0
\end{array}\right) \hat{\alpha}_{t \mid t}
$$

This gives a Kalman filter prediction that can be written as

$$
E_{K F}\left(y_{t+h} \mid I_{t}^{M}\right)=\sum_{j=0}^{\infty} w_{y, j}^{K F} y_{t-j}+\sum_{j=0}^{\infty} w_{x, j}^{K F} x_{t-j / m}
$$

As in the previous subsection, we can find the regular or multiplicative MIDAS parameters that get as close as possible to the Kalman filter using the objective function, given in equation (3.6). In this two-factor model, the elements of $\Sigma_{x y}$, the variance-covariance matrix 
of $\left(x_{t}, y_{t}^{*}, x_{t-1 / m}, y_{t-1 / m}^{*}, \ldots . x_{t-\bar{K}}, y_{t-\bar{K}}^{*}\right)^{\prime}$, are as follows:

$$
\begin{aligned}
& \operatorname{Cov}\left(y_{t-i / m}^{*}, y_{t-j / m}^{*}\right)=\gamma_{1,1}^{2} \frac{\rho_{1}^{|i-j|} \sigma_{\eta, 1}^{2}}{1-\rho_{1}^{2}}+\gamma_{1,2}^{2} \frac{\rho_{2}^{|i-j|} \sigma_{\eta, 2}^{2}}{1-\rho_{2}^{2}}+\frac{d_{1}^{|i-j|} \sigma_{y}^{2}}{1-d_{1}^{2}} \\
& \operatorname{Cov}\left(x_{t-i / m}, x_{t-j / m}\right)=\gamma_{2,1}^{2} \frac{\rho_{1}^{|i-j|} \sigma_{\eta, 1}^{2}}{1-\rho_{1}^{2}}+\gamma_{2,2}^{2} \frac{\rho_{2}^{|i-j|} \sigma_{\eta, 2}^{2}}{1-\rho_{2}^{2}}+\frac{d_{2}^{|i-j|} \sigma_{x}^{2}}{1-d_{2}^{2}} \\
& \operatorname{Cov}\left(x_{t-i / m}, y_{t-j / m}^{*}\right)=2 \gamma_{1,1} \gamma_{2,1} \frac{\rho_{1}^{|i-j|} \sigma_{\eta, 1}^{2}}{1-\rho_{1}^{2}}+2 \gamma_{1,2} \gamma_{2,2} \frac{\rho_{2}^{|i-j|} \sigma_{\eta, 2}^{2}}{1-\rho_{2}^{2}}
\end{aligned}
$$

for $i, j=0,1,2, \ldots 3 \bar{K}$, where $\sigma_{\eta, 1}^{2}=\operatorname{Var}\left(\eta_{1, t}\right), \sigma_{\eta, 2}^{2}=\operatorname{Var}\left(\eta_{2, t}\right), \sigma_{y}^{2}=\operatorname{Var}\left(\varepsilon_{1, t}\right)$ and $\sigma_{x}^{2}=\operatorname{Var}\left(\varepsilon_{2, t}\right)$.

Panel A of Table 2 shows again the minimized values of the $\mathbb{L}^{2}$ objective function comparing Kalman Filter and MIDAS regressions (regular and multiplicative), with $d=d_{1}=d_{2}, \gamma_{1}=$ $\gamma_{2}=1$ and $\sigma_{\eta}^{2}=\sigma_{y}^{2}=\sigma_{x}^{2}=1$. Results are shown for combinations of $d$ and $\rho$ and the forecast horizon, $h=2$ and 4 . In Panel $\mathrm{B}$ we also again report the $\mathbb{L}^{2}$ values that correspond to the minimized prediction errors. We do not report the results for the prediction error ratios as they are again easy to summarize - for all combinations of $d$ and $\rho$ the predictions are for all practical purposes equal, i.e. the value of PE-ratio is numerically extremely close to one.

Overall the results in Table 2 are quite similar to those in Table 1 and show that MIDAS provides a good general fit to the Kalman filter weights. There are however a few differences with the results for the one-factor case. First, for $d=0$ and $\rho>0$, multiplicative MIDAS is no longer a perfect fit to the Kalman Filter. Yet, we see that it is for all practical purposes, as is the regular MIDAS specification. Second, differences between the multiplicative and regular specifications for the extremes are smaller than in the one-factor case considered in Table 1 , especially in the case of $\rho=0.99$. Third, the entries to Panel A of Table 2 are essentially all zero. Hence, according distance criteria, the differences are very small. Finally, as in Table 1, we find the impact of the forecast horizon to be negligible.

\subsection{Specification Errors}

All the models considered so far are correctly specified, and so the MIDAS regression cannot hope to do better than the Kalman filter, in population at least. However, this is not true any more if the state space model is mis-specified. Accordingly in this section, we consider the 
case in which the Kalman filter weights are computed assuming that the data are generated by a one-factor model, whereas in fact the data are generated by a two-factor model. The MIDAS regressions are selected so as to approximate the data generating process minimizing the objective function (3.6) from a two-factor model.

We consider two MIDAS specifications as before: regular and multiplicative. In terms of parameter configurations for the two-factor model appearing in subsection 3.2 we consider two experiments. The first involves $\rho_{1}=\rho_{2}=\rho$ and $d_{1}=d_{2}=d$. The values taken by $d$ and $\rho$ are the same as before, and the forecast horizon is $h$. The results appear in Table 3, for the regular (Panel A) and multiplicative (Panel B) MIDAS regressions, respectively. The second experiment sets $\rho_{1} \neq \rho_{2}, d_{1}=d_{2}=0$ (so that the measurement noise is i.i.d.). The results appear in Table 4. In contrast to Tables 1 and 2, we do have nontrivial differences between the prediction errors. Hence, we report the ratio of prediction errors appearing in equation (3.7) and cover again two forecast horizons $h=2,4$. In the interest of saving space we do not report the corresponding minimized values of the $\mathbb{L}^{2}$. They are available upon request. Since the ratios are $P E-M I D A S$ divided by $P E-S S 1$, values below one imply that MIDAS provides better predictions than the mis-specified Kalman filter. The results in the two tables are quite remarkable. If we take away the extremes, especially $\rho=0.99$, it turns out that the MIDAS regressions are almost always better predictors. On average, the gains range between $10 \%$ and $20 \%$, although ratios as low as 0.58 can be reached.

One particularly interesting panel is the upper left one in Table 4 , which covers $\rho_{1} \neq \rho_{2}, d_{1}$ $=d_{2}=0$ for $h=2$ and the regular MIDAS specification. In this case none of the entries are above one. This means that in all cases considered, regular MIDAS outperforms the mis-specified Kalman filter. For the one-year horizon $(h=4)$ this appears almost true too, except in the few cases where the Kalman filter only does slightly better. The multiplicative specification does not fare as well, particularly at the extreme cases, at the short horizon $h$ $=2$. At the longer horizon the differences between the two MIDAS specifications in Table 4 appear minor, however.

\section{Empirical Study}

As an illustration of the theoretical results in sections 2 and 3, we present an empirical application to forecasting of U.S. GDP growth. In a first subsection we describe the data. 
The results are discussed in a second subsection.

\subsection{The Data}

We use a dataset with mixed frequencies, monthly and quarterly. The variable to be predicted is the growth rate of real GDP from 1959Q1 to 2009Q1. The explanatory variables include nine monthly indicators until May 2009. In particular, we consider the term spread (TERM), stock market returns (SP500), industrial production (IP), employment (Emply), consumer expectations (Exptn), personal income (PI), the leading index (LEI), manufacturing (Manu), and oil prices (Oil). They are transformed to induce stationarity and to insure that the transformed variables correspond to the real GDP growth observed at the end of the quarter. See Table 5 for more details on the definition and data transformations. ${ }^{2}$ It should also be noted that we focus exclusively on one-factor state space models.

Each model uses just one out of nine monthly indicators. The forecasts are in all cases made using monthly data up to and including the second month of the quarter. We evaluate the state space and MIDAS forecasts in a standard recursive prediction exercise. The first estimation window is from 1959:Q1 to 1978:Q4, and is recursively expanded over time. For example, for MIDAS, a one-step-ahead forecast of 1979:Q1 is generated from regressing GDP growth up to 1978:Q4 on its own lags and the monthly predictor up to 1978:11 (November). Then the values of GDP growth through 1978:Q4 and of the monthly predictor up to 1979:02 (February) are used with the estimated coefficients to predict the 1979:Q1 GDP growth rate. We also do two- to eight-quarter-ahead forecasting in a similar fashion. The evaluation sample is from 1979:Q1 to 2009Q1. Some monthly predictors are available only for more recent subsamples (e.g. crude oil price and manufacturing). In these cases, we use the first 40 quarters as the estimation sample and the remaining period until 2009Q1 as the evaluation sample. We should also note that - as usually is done in the context of state space models, all series are normalized by the (full sample) mean and variance.

In line with Kuzin, Marcellino, and Schumacher (2009), we specify the lag order in the mixed-frequency state space model by applying the Bayesian information criterion (BIC) with a maximum lag order of $p=4$ months. We also find that the chosen lag lengths are

\footnotetext{
${ }^{2}$ Note that, because real-time vintages for all the series in the panel are not available, we did not perform a pure real-time forecasting exercise. Authors such as Bernanke and Boivin (2003) and Schumacher and Breitung (2008) find that data revisions have limited impact on forecasting accuracy for economic activity.
} 
usually small with only one or two lags in most cases. In both the regular and multiplicative MIDAS model, we set the maximum number of lags as $K_{y}=1$ and $K_{x}=6$ quarters and choose the lag length by the minimum in-sample fitting error criterion. Finally, we use the root mean squared forecasting error (RMSE) to evaluate each model's forecasting accuracy:

$$
R M S E(h)=\sqrt{\frac{1}{T_{1}-T_{2}-h+1} \sum_{t=T_{1}}^{T_{2}-h}\left(\widehat{Y}_{t+h}-Y_{t+h}\right)^{2}},
$$

where the model is estimated for the period of $t=\left[1, T_{1}\right]$, and the forecasting period is given by $t=\left[T_{1}+h, T_{2}\right]$.

\subsection{Forecasting Results}

Table 6 compares the forecasting performance between the regular MIDAS, multiplicative MIDAS and state space models. We consider horizons from one quarter up to two years. Recall that all the series are normalized by the (full sample) mean and variance, including real GDP growth. So the root mean squared forecasting errors reported in Table 6 are in standard deviation units. We report the level of root mean squared forecasting errors for state space models (denoted m0), and for regular MIDAS (denoted m1) and multiplicative MIDAS (denoted $\mathrm{m} 2)$. In addition, we also report the ratios $(\mathrm{m} 0 / \mathrm{m} 1)$ and $(\mathrm{m} 0 / \mathrm{m} 2)$. When we see entries for ratios of say 0.80 , we can interpret this as gains equivalent to $20 \%$ of the full sample standard deviation of GDP growth. The ratios above one imply that MIDAS regressions produce better forecasts. Conversely, ratios below one imply that the Kalman filter produces better forecasts.

When we consider the various series reported in Table 6, we see that MIDAS gives better forecasts when the term spread and consumer expectations are used as predictors. On the other hand, for the personal income and manufacturing series, the Kalman filter dominates at all horizons. For the other series the results are mixed, with ratios generally slightly above or below one. The results also differ across horizons, without a clear pattern. At the longest horizon $(h=8)$, except for term spread and consumer expectations, we note a slight preference for the Kalman filter - although the ratios are typically within a 5 to $10 \%$ range. Overall, the results support the theoretical deduction obtained in the previous section. In some cases MIDAS clearly outperforms the state space approach, perhaps because the model 
is mis-specified. In other cases, the Kalman filter performs well, but the MIDAS model does too, and there is often little difference between them.

To conclude it is worth summarizing the Table 6 across all series - and by doing so, we observe the best predictor with the regular/multiplicative MIDAS and state space models is the crude oil price, except at the longest horizons.

\begin{tabular}{clccccccccc}
\multicolumn{1}{c}{ h (Quarter) } & 1 & 2 & 3 & 4 & 5 & 6 & 7 & 8 \\
\hline \multirow{2}{*}{ Best } & State Space & Oil & Oil & Oil & Oil & Oil & Oil & LEI & LEI \\
Predictor & Regular MIDAS & Oil & Oil & Oil & Oil & Oil & LEI & Emply & Emply \\
& Multiplicative MIDAS & Oil & Oil & Oil & Oil & Oil & Term & Emply & IP \\
\hline \multirow{5}{*}{ RMSE } & State Space & 0.69 & 0.65 & 0.68 & 0.67 & 0.70 & 0.70 & 0.74 & 0.76 \\
& Regular MIDAS & 0.65 & 0.76 & 0.70 & 0.74 & 0.72 & 0.78 & 0.80 & 0.79 \\
& Multiplicative MIDAS & 0.65 & 0.77 & 0.72 & 0.76 & 0.70 & 0.78 & 0.80 & 0.79
\end{tabular}

When we look at the best performance series in the above table we find evidence similar to Kuzin, Marcellino, and Schumacher (2009) - they find gains at short horizons from using MIDAS and the reverse for longer horizons (two years, as in our application). For intermediate horizons we find the Kalman filter to be best. Overall, however the differences are often small.

\section{Conclusion}

We examined the relationship between MIDAS regressions and Kalman filter state space models applied to mixed frequency data. State space models consist of a system of two equations, a measurement equation which links observed series to a latent state process, and a state equation which describes the state process dynamics. The system of equations therefore typically requires a lot of parameters, for the measurement equation, the state dynamics and their error processes. In contrast, recent work by Ghysels, Santa-Clara, and Valkanov (2002), Ghysels, Santa-Clara, and Valkanov (2006) and Andreou, Ghysels, and Kourtellos (2008a) using MIDAS regressions handles mixed sample frequencies in a simple 
single equation setting that is easy to estimate. We showed that MIDAS regressions and the Kalman steady state linear filter can be identical - and if they are not - the former is very close in terms of prediction behavior. One advantage of MIDAS regressions is that they are less prone to specifications errors. In fact, we show that the latter can impair Kalman filter predictions.

Finally, it is important to note that estimating Kalman filter specifications is numerically much more involved. In contrast, all MIDAS estimations are in comparison computationally simple. This is relevant as the computational complexity limits the applicability of the Kalman filter to a small set of series. For example Aruoba, Diebold, and Scotti (2009) construct a very useful Business Conditions Index published in real time by the Federal Reserve Bank of Philadelphia. The index is based on a small set of series sampled at mixed frequencies (weekly initial jobless claims; monthly payroll employment, industrial production, personal income less transfer payments, manufacturing and trade sales; and quarterly real GDP). In contrast, Andreou, Ghysels, and Kourtellos (2008b) compute macro economic forecasts with MIDAS regressions using close to a hundred daily financial series which they combine via Bayesian model averaging. This is a fairly straightforward exercise with MIDAS regressions, but would be computationally very difficult with a fully specified state space model. 


\section{References}

Alper, C.E., S. Fendoglu, and B. Saltoglu, 2008, Forecasting Stock Market Volatilities Using MIDAS Regressions: An Application to the Emerging Markets, MPRA Paper No. 7460.

Andreou, E., E. Ghysels, and A. Kourtellos, 2008a, Regression Models With Mixed Sampling Frequencies, Journal of Econometrics, forthcoming.

— , 2008b, Should macroeconomic forecasters look at daily financial data?, Discussion paper, Discussion Paper UNC and University of Cyprus.

Armesto, M.T., R. Hernandez-Murillo, M. Owyang, and J. Piger, 2008, Measuring the Information Content of the Beige Book: A Mixed Data Sampling Approach, Journal of Money, Credit and Banking (forthcoming).

Aruoba, S., F. Diebold, and C. Scotti, 2009, Real time measurement of business conditions, Journal of Business and Economic Statistics 27, 417-427.

Assimakis, N., and M. Adam, 2009, Steady State Kalman Filter for Periodic Models: A New Approach, International Journal of Contemporary Mathematical Sciences 4, 201-218.

Bai, J., and S. Ng, 2004, A PANIC attack on unit roots and cointegration, Econometrica pp. $1127-1177$.

Bernanke, B.S., and J. Boivin, 2003, Monetary policy in a data-rich environment, Journal of Monetary Economics 50, 525-546.

Bernanke, Ben, Mark Gertler, and Mark Watson, 1997, Systematic monetary policy and the effects of oil price shocks, Brookings Papers on Economic Activity 1, 91-157.

Chen, X., and E. Ghysels, 2009, News-good or bad-and its impact on volatility predictions over multiple horizons, Discussion Paper, UNC.

Clements, M.P., and A.B. Galvão, 2008a, Forecasting US output growth using Leading Indicators: An appraisal using MIDAS models, Journal of Applied Econometrics (forthcoming).

Clements, M., and A. Galvão, 2008b, Macroeconomic Forecasting with Mixed Frequency Data: Forecasting US output growth, Journal of Business and Economic Statistics 26, $546-554$. 
Engle, R.F., E. Ghysels, and B. Sohn, 2008, On the Economic Sources of Stock Market Volatility, Discussion Paper NYU and UNC.

Forni, M., M. Hallin, M. Lippi, and L. Reichlin, 2000, The generalized dynamic-factor model: Identification and estimation, Review of Economics and Statistics 82, 540-554.

Forsberg, L., and E. Ghysels, 2006, Why do absolute returns predict volatility so well?, Journal of Financial Econometrics 6, 31-67.

Galvão, A.B., 2006, Changes in Predictive Ability with Mixed Frequency Data, Discussion Paper QUeen Mary.

Ghysels, Eric, Pedro Santa-Clara, and Rossen Valkanov, 2002, The MIDAS touch: Mixed data sampling regression models, Working paper, UNC and UCLA.

— 2005, There is a risk-return tradeoff after all, Journal of Financial Economics 76, $509-548$.

— 2006, Predicting volatility: getting the most out of return data sampled at different frequencies, Journal of Econometrics 131, 59-95.

Ghysels, Eric, and Jonathan Wright, 2009, Forecasting professional forecasters, Journal of Business and Economic Statistics 27, 504-516.

Hamilton, J.D., 2006, Daily Monetary Policy Shocks and the Delayed Response of New Home Sales, working paper, UCSD.

Harvey, Andrew, 1989, Forecasting, Structural Time Series Models and the Kalman Filter (Cambridge University Press, Cambridge).

Harvey, Andrew C., and Richard G. Pierse, 1984, Estimating missing observations in economic time series, Journal of the American Statistical Association 79, 125-131.

Kuzin, V., M. Marcellino, and C. Schumacher, 2009, MIDAS versus mixed-frequency VAR: nowcasting GDP in the euro area, Discussion Paper 07/2009 Deutsche Bundesbank.

León, Á., J.M. Nave, and G. Rubio, 2007, The relationship between risk and expected return in Europe, Journal of Banking and Finance 31, 495-512.

Mariano, R.S., and Y. Murasawa, 2003, A new coincident index of business cycles based on monthly and quarterly series, Journal of Applied Econometrics 18, 427-443. 
Mittnik, S., and P. Zadrozny, 2004, Forecasting quarterly German GDP at monthly intervals using monthly Ifo business conditions data (Springer).

Nunes, L.C., 2005, Nowcasting quarterly GDP growth in a monthly coincident indicator model, Journal of Forecasting 24, 575.

Schumacher, C., and J. Breitung, 2008, Real-time forecasting of German GDP based on a large factor model with monthly and quarterly data, International Journal of Forecasting $24,386-398$.

Stock, J.H., and M.W. Watson, 1989, New indexes of coincident and leading economic indicators, NBER macroeconomics annual pp. 351-394.

— , 2002, Macroeconomic forecasting using diffusion indexes, Journal of Business and Economic Statistics 20, 147-162.

Tay, A., 2006, Financial Variables as Predictors of Real Output Growth, Discussion Paper SMU.

Tay, A.S., 2007, Mixing Frequencies: Stock Returns as a Predictor of Real Output Growth, Discussion Paper SMU.

Zadrozny, P.A., 1990, Forecasting US GNP at monthly intervals with an estimated bivariate time series model, Federal Reserve Bank of Atlanta Economic Review 75, 2-15. 
Technical Appendices

\section{A One-Factor State Space Model with Correlated Measurement Errors}

We start from the state space model appearing in subsection 3.1 repeated here for convenience:

$$
\begin{array}{llll}
f_{t+j / m}=\rho f_{t+(j-1) / m}+\eta_{t+j / m} & \forall t & j=0, \ldots, m-1 \\
y_{t+j / m}^{*}=\gamma_{1} f_{t+j / m}+u_{1, t+j / m} & \forall t & j=0, \ldots, m-1 \\
x_{t+j / m}=\gamma_{2} f_{t+j / m}+u_{2, t+j / m} & \forall t & j=0, \ldots, m-1
\end{array}
$$

where

$$
u_{i, t+j / m}-d_{i} u_{i, t+(j-1) / m}=\epsilon_{i, t+j / m} \quad i=1,2 .
$$

with periodic Kalman gain matrices:

$$
K_{1 \mid 0}=\left(\begin{array}{c}
\kappa_{1}^{1} \\
\kappa_{2}^{1} \\
\kappa_{3}^{1}
\end{array}\right), K_{2 \mid 1}=\left(\begin{array}{c}
\kappa_{1}^{2} \\
\kappa_{2}^{2} \\
\kappa_{3}^{2}
\end{array}\right) \text { and } K_{3 \mid 2}=\left(\begin{array}{cc}
\kappa_{1,1}^{3} & \kappa_{1,2}^{3} \\
\kappa_{2,1}^{3} & \kappa_{2,2}^{3} \\
\kappa_{3,1}^{3} & \kappa_{3,2}^{3}
\end{array}\right)
$$

As noted in section 3.1 , the state vector is $\alpha_{t+j / m}=\left(f_{t+j / m}, u_{1, t+j / m}, u_{2, t+j / m}\right)^{\prime}$ and we have

$$
\begin{gathered}
F=\left(\begin{array}{ccc}
\rho & 0 & 0 \\
0 & d_{1} & 0 \\
0 & 0 & d_{2}
\end{array}\right), \\
Z_{j}=\left(\begin{array}{ccc}
\gamma_{2} & 0 & 1
\end{array}\right) \text { for } 0<j \leq m-1 \text { and } Z_{0}=\left(\begin{array}{ccc}
\gamma_{1} & 1 & 0 \\
\gamma_{2} & 0 & 1
\end{array}\right) .
\end{gathered}
$$

Using the formula $A_{j \mid j-1}=F-K_{j \mid j-1} Z_{j} F$, we can write

$$
A_{1 \mid 0}=\left(\begin{array}{ccc}
\rho-\rho \gamma_{2} \kappa_{1}^{1} & 0 & -\kappa_{1}^{1} d_{2} \\
-\rho \gamma_{2} \kappa_{2}^{1} & d_{1} & -\kappa_{2}^{1} d_{2} \\
-\rho \gamma_{2} \kappa_{3}^{1} & 0 & d_{2}-\kappa_{3}^{1} d_{2}
\end{array}\right)
$$




$$
A_{2 \mid 1}=\left(\begin{array}{ccc}
\rho-\rho \gamma_{2} \kappa_{1}^{2} & 0 & -\kappa_{1}^{2} d_{2} \\
-\rho \gamma_{2} \kappa_{2}^{2} & d_{1} & -\kappa_{2}^{2} d_{2} \\
-\rho \gamma_{2} \kappa_{3}^{2} & 0 & d_{2}-\kappa_{3}^{2} d_{2}
\end{array}\right)
$$

and

$$
A_{3 \mid 2}=\left(\begin{array}{ccc}
\rho-\rho\left(\gamma_{1} \kappa_{1,1}^{3}+\gamma_{2} \kappa_{1,2}^{3}\right) & -\kappa_{1,1}^{3} d_{1} & -\kappa_{1,2}^{3} d_{2} \\
-\rho\left(\gamma_{1} \kappa_{2,1}^{3}+\gamma_{2} \kappa_{2,2}^{3}\right) & d_{1}-\kappa_{2,1}^{3} d_{1} & -\kappa_{2,2}^{3} d_{2} \\
-\rho\left(\gamma_{1} \kappa_{3,1}^{3}+\gamma_{2} \kappa_{3,2}^{3}\right) & -\kappa_{3,1}^{3} d_{1} & d_{2}-\kappa_{3,2}^{3} d_{2}
\end{array}\right)
$$

Letting $\tilde{A}_{3}^{m}=A_{3 \mid 2}, \tilde{A}_{2}^{m}=A_{3 \mid 2} A_{2 \mid 1}$ and $\tilde{A}_{1}^{m}=A_{3 \mid 2} A_{2 \mid 1} A_{1 \mid 0}$ as before, and adopting the notation that $[A]_{i j}$ refers to the $i j$ th element of the matrix $A$, from equation (2.13), the Kalman filter implies the following equation for $h$-quarter-ahead prediction:

$$
\begin{aligned}
E_{K F}\left[y_{t+h} \mid I_{t}^{M}\right]= & E\left(\gamma_{1} f_{t+h}+u_{1, t+h} \mid I_{t}^{M}\right)=\gamma_{1} \rho^{3 h} E\left(f_{t} \mid I_{t}^{M}\right)+d_{1}^{3 h} E\left(u_{1, t} \mid I_{t}^{M}\right) \\
= & \gamma_{1} \rho^{3 h} \sum_{j=0}^{\infty}\left\{\left[\left(\tilde{A}_{1}^{m}\right)^{j}\right]_{11} \kappa_{1,1}^{3}+\left[\left(\tilde{A}_{1}^{m}\right)^{j}\right]_{12} \kappa_{2,1}^{3}+\left[\left(\tilde{A}_{1}^{m}\right)^{j}\right]_{13} \kappa_{3,1}^{3}\right\} y_{t-j} \\
& +\gamma_{1} \rho^{3 h} \sum_{j=0}^{\infty}\left\{\left[\left(\tilde{A}_{1}^{m}\right)^{j}\right]_{11} \kappa_{1,2}^{3}+\left[\left(\tilde{A}_{1}^{m}\right)^{j}\right]_{12} \kappa_{2,2}^{3}+\left[\left(\tilde{A}_{1}^{m}\right)^{j}\right]_{13} \kappa_{3,2}^{3}\right\} x_{t-j} \\
& +\gamma_{1} \rho^{3 h} \sum_{j=0}^{\infty}\left\{\left[\left(\tilde{A}_{1}^{m}\right)^{j} \tilde{A}_{2}^{m}\right]_{11} \kappa_{1}^{2}+\left[\left(\tilde{A}_{1}^{m}\right)^{j} \tilde{A}_{2}^{m}\right]_{12} \kappa_{2}^{2}+\left[\left(\tilde{A}_{1}^{m}\right)^{j} \tilde{A}_{2}^{m}\right]_{13} \kappa_{3}^{2}\right\} x_{t-j-1 / 3} \\
& +\gamma_{1} \rho^{3 h} \sum_{j=0}^{\infty}\left\{\left[\left(\tilde{A}_{1}^{m}\right)^{j} \tilde{A}_{3}^{m}\right]_{11} \kappa_{1}^{1}+\left[\left(\tilde{A}_{1}^{m}\right)^{j} \tilde{A}_{3}^{m}\right]_{12} \kappa_{2}^{1}+\left[\left(\tilde{A}_{1}^{m}\right)^{j} \tilde{A}_{3}^{m}\right]_{13} \kappa_{3}^{1}\right\} x_{t-j-2 / 3} \\
& +d_{1}^{3 h} \sum_{j=0}^{\infty}\left\{\left[\left(\tilde{A}_{1}^{m}\right)^{j}\right]_{21} \kappa_{1,1}^{3}+\left[\left(\tilde{A}_{1}^{m}\right)^{j}\right]_{22} \kappa_{2,1}^{3}+\left[\left(\tilde{A}_{1}^{m}\right)^{j}\right]_{23} \kappa_{3,1}^{3}\right\} y_{t-j} \\
& +d_{1}^{3 h} \sum_{j=0}^{\infty}\left\{\left[\left(\tilde{A}_{1}^{m}\right)^{j}\right]_{21} \kappa_{1,2}^{3}+\left[\left(\tilde{A}_{1}^{m}\right)^{j}\right]_{22} \kappa_{2,2}^{3}+\left[\left(\tilde{A}_{1}^{m}\right)^{j}\right]_{23} \kappa_{3,2}^{3}\right\} x_{t-j} \\
& +d_{1}^{3 h} \sum_{j=0}^{\infty}\left\{\left[\left(\tilde{A}_{1}^{m}\right)^{j} \tilde{A}_{2}^{m}\right]_{21} \kappa_{1}^{2}+\left[\left(\tilde{A}_{1}^{m}\right)^{j} \tilde{A}_{2}^{m}\right]_{22} \kappa_{2}^{2}+\left[\left(\tilde{A}_{1}^{m}\right)^{j} \tilde{A}_{2}^{m}\right]_{23} \kappa_{3}^{2}\right\} x_{t-j-1 / 3} \\
& +d_{1}^{3 h} \sum_{j=0}^{\infty}\left\{\left[\left(\tilde{A}_{1}^{m}\right)^{j} \tilde{A}_{3}^{m}\right]_{21} \kappa_{1}^{1}+\left[\left(\tilde{A}_{1}^{m}\right)^{j} \tilde{A}_{3}^{m}\right]_{22} \kappa_{2}^{1}+\left[\left(\tilde{A}_{1}^{m}\right)^{j} \tilde{A}_{3}^{m}\right]_{23} \kappa_{3}^{1}\right\} x_{t-j-2 / 3}(\mathrm{~A} .4)
\end{aligned}
$$

As noted in section 3.1, the variance of the $h$-quarter-ahead Kalman Filter forecast errors is 
$w_{K F}^{\prime} \Sigma_{x y} w_{K F}$ where

$$
\begin{aligned}
w_{K F}= & \left(1,0_{m h-1 \times 1},-w_{x, 1}^{K F},-w_{y, 1}^{K F},-w_{x, 2}^{K F}, 0,-w_{x, 3}^{K F}, 0,\right. \\
& \left.-w_{x, 4}^{K F},-w_{y, 2}^{K F},-w_{x, 5}^{K F}, 0,-w_{x, 6}^{K F}, 0, \ldots-w_{x, 3 \bar{K}-2}^{K F},-w_{y, \bar{K}}^{K F},-w_{x, 3 \bar{K}-1}^{K F}, 0,-w_{x, 3 \bar{K}}^{K F}, 0\right)^{\prime}
\end{aligned}
$$

Similarly, the variance of the corresponding MIDAS forecast errors is $w_{M d s}^{\prime} \Sigma_{x y} w_{M d s}$ where

$$
\begin{aligned}
w_{M d s}= & \left(1,0_{m h-1 \times 1},-w_{x, 1}^{M d s},-w_{y, 1}^{M d s},-w_{x, 2}^{M d s}, 0,-w_{x, 3}^{M d s}, 0\right. \\
& \left.-w_{x, 4}^{M d s},-w_{y, 2}^{M d s},-w_{x, 5}^{M d s}, 0,-w_{x, 6}^{M d s}, 0, \ldots-w_{x, 3 \bar{K}-2}^{M d s},-w_{y, \bar{K}}^{M d s},-w_{x, 3 \bar{K}-1}^{M d s}, 0,-w_{x, 3 \bar{K}}^{M d s}, 0\right)^{\prime}
\end{aligned}
$$

\section{B Two-Factor State Space Model with Correlated Measurement Errors}

We consider a two-factor state space model appearing in section 3.2 repeated here for convenience:

$$
\begin{aligned}
F_{t+j / m} & =\left(\begin{array}{l}
f_{1, t+j / m} \\
f_{2, t+j / m}
\end{array}\right)=\left(\begin{array}{cc}
\rho_{1} & 0 \\
0 & \rho_{2}
\end{array}\right)\left(\begin{array}{c}
f_{1, t+(j-1) / m} \\
f_{2, t+(j-1) / m}
\end{array}\right)+\left(\begin{array}{l}
\eta_{1, t+j / m} \\
\eta_{2, t+j / m}
\end{array}\right) \quad j=0, \ldots, m-1 \\
y_{t+j / m}^{*} & =\gamma_{1}^{\prime} F_{t+j / m}+u_{1, t+j / m} \quad \forall t \quad j=0, \ldots, m-1 \\
x_{2, t+j / m} & =\gamma_{2}^{\prime} F_{t+j / m}+u_{2, t+j / m} \quad \forall t \quad j=0, \ldots, m-1
\end{aligned}
$$

where

$$
u_{i, t+j / m}-d_{i} u_{i, t+(j-1) / m}=\epsilon_{i, t+j / m} \quad i=1,2 .
$$

Then the periodic Kalman gain matrices are:

$$
K_{1 \mid 0}=\left(\begin{array}{c}
\kappa_{1}^{1} \\
\kappa_{2}^{1} \\
\kappa_{3}^{1} \\
\kappa_{4}^{1}
\end{array}\right), K_{2 \mid 1}=\left(\begin{array}{c}
\kappa_{1}^{2} \\
\kappa_{2}^{2} \\
\kappa_{3}^{2} \\
\kappa_{4}^{2}
\end{array}\right) \text { and } K_{3 \mid 1}=\left(\begin{array}{cc}
\kappa_{1,1}^{3} & \kappa_{1,2}^{3} \\
\kappa_{2,1}^{3} & \kappa_{2,2}^{3} \\
\kappa_{3,1}^{3} & \kappa_{3,2}^{3} \\
\kappa_{4,1}^{3} & \kappa_{4,2}^{3}
\end{array}\right)
$$

The state vector is $\alpha_{t+j / m}=\left(f_{1, t+j / m}, f_{2, t+j / m}, u_{1, t+j / m}, u_{2, t+j / m}\right)^{\prime}$ and we have $F=$ 
$\left(\begin{array}{cccc}\rho_{1} & 0 & 0 & 0 \\ 0 & \rho_{2} & 0 & 0 \\ 0 & 0 & d_{1} & 0 \\ 0 & 0 & 0 & d_{2}\end{array}\right), Z_{j}=\left(\begin{array}{llll}\gamma_{2,1} & \gamma_{2,2} & 0 & 1\end{array}\right)$ and $Z_{0}=\left(\begin{array}{cccc}\gamma_{1,1} & \gamma_{1,2} & 1 & 0 \\ \gamma_{2,1} & \gamma_{2,2} & 0 & 1\end{array}\right)$. Hence, since
$A_{j \mid j-1}=F-K_{j \mid j-1} Z_{j} F$, we can write

$$
\begin{aligned}
A_{1 \mid 0} & =\left(\begin{array}{cccc}
\rho_{1}-\rho_{1} \gamma_{2,1} \kappa_{1}^{1} & -\rho_{2} \gamma_{2,2} \kappa_{1}^{1} & 0 & -\kappa_{1}^{1} d_{2} \\
-\rho_{1} \gamma_{2,1} \kappa_{2}^{1} & \rho_{2}-\rho_{2} \gamma_{2,2} \kappa_{2}^{1} & 0 & -\kappa_{2}^{1} d_{2} \\
-\rho_{1} \gamma_{2,1} \kappa_{3}^{1} & -\rho_{2} \gamma_{2,2} \kappa_{3}^{1} & d_{1} & -\kappa_{3}^{1} d_{2} \\
-\rho_{1} \gamma_{2,1} \kappa_{4}^{1} & -\rho_{2} \gamma_{2,2} \kappa_{4}^{1} & 0 & d_{2}-\kappa_{4}^{1} d_{2}
\end{array}\right) \\
A_{2 \mid 1} & =\left(\begin{array}{cccc}
\rho_{1}-\rho_{1} \gamma_{2,1} \kappa_{1}^{1} & -\rho_{2} \gamma_{2,2} \kappa_{1}^{1} & 0 & -\kappa_{1}^{1} d_{2} \\
-\rho_{1} \gamma_{2,1} \kappa_{2}^{1} & \rho_{2}-\rho_{2} \gamma_{2,2} \kappa_{2}^{1} & 0 & -\kappa_{2}^{1} d_{2} \\
-\rho_{1} \gamma_{2,1} \kappa_{3}^{1} & -\rho_{2} \gamma_{2,2} \kappa_{3}^{1} & d_{1} & -\kappa_{3}^{1} d_{2} \\
-\rho_{1} \gamma_{2,1} \kappa_{4}^{1} & -\rho_{2} \gamma_{2,2} \kappa_{4}^{1} & 0 & d_{2}-\kappa_{4}^{1} d_{2}
\end{array}\right)
\end{aligned}
$$

and

$$
A_{3 \mid 2}=\left(\begin{array}{cccc}
\rho_{1}-\rho_{1}\left(\gamma_{1,1} \kappa_{1,1}^{3}+\gamma_{2,1} \kappa_{1,2}^{3}\right) & -\rho_{2}\left(\gamma_{1,2} \kappa_{1,1}^{3}+\gamma_{2,2} \kappa_{1,2}^{3}\right) & -\kappa_{1,1}^{3} d_{1} & -\kappa_{1,1}^{3} d_{2} \\
-\rho_{1}\left(\gamma_{1,1} \kappa_{2,1}^{3}+\gamma_{2,1} \kappa_{2,2}^{3}\right) & \rho_{2}-\rho_{2}\left(\gamma_{1,2} \kappa_{2,1}^{3}+\gamma_{2,2} \kappa_{2,2}^{3}\right) & -\kappa_{2,1}^{3} d_{1} & -\kappa_{2,1}^{3} d_{2} \\
-\rho_{1}\left(\gamma_{1,1} \kappa_{3,1}^{3}+\gamma_{2,1} \kappa_{3,2}^{3}\right) & -\rho_{2}\left(\gamma_{1,2} \kappa_{3,1}^{3}+\gamma_{2,2} \kappa_{3,2}^{3}\right) & d_{1}-\kappa_{3,1}^{3} d_{1} & -\kappa_{3,1}^{3} d_{2} \\
-\rho_{1}\left(\gamma_{1,1} \kappa_{4,1}^{3}+\gamma_{2,1} \kappa_{4,2}^{3}\right) & -\rho_{2}\left(\gamma_{1,2} \kappa_{4,1}^{3}+\gamma_{2,2} \kappa_{4,2}^{3}\right) & -\kappa_{4,1}^{3} d_{1} & d_{2}-\kappa_{4,1}^{3} d_{2}
\end{array}\right)
$$




\section{Table 1: Approximation Results between MIDAS and One-Factor State Space Model}

Entries in Panel A pertain to the values of the $L^{2}$ measure appearing in equation (3.8) comparing Kalman Filter for the one-factor model appearing in equations (3.1) and (3.2). In Panel B the entries pertain to the values of the $L^{2}$ when the MIDAS weights are optimized in terms of minimal prediction error - as appearing in equation (3.6). Entries cover both regular and multiplicative MIDAS regressions, with $d=d_{1}$ $=d_{2}, \gamma_{1}=\gamma_{2}=1$ and $\sigma_{\text {eta }}^{2}=\sigma_{y}^{2}=\sigma_{x}^{2}=1$. The multiplicative MIDAS scheme refers to the ADL-MIDAS regression appearing in equation (2.23), whereas the regular one refers to equation (3.5).

Panel A: $\mathbb{L}^{2}$ distance

Regular MIDAS

Multiplicative MIDAS

\begin{tabular}{rlllllllllllllllll}
\multicolumn{11}{c}{ Regular MIDAS } \\
\hline \multicolumn{11}{c|}{ Forecasting Horizon: Two Quarters Ahead $(\mathrm{h}=2)$} \\
$d \backslash \rho$ & -0.9 & -0.5 & 0 & 0.5 & 0.9 & 0.95 & 0.99 & -0.9 & -0.5 & 0 & 0.5 & 0.9 & 0.95 & 0.99 \\
\hline-0.9 & 0.00 & 0.00 & 0.01 & 0.01 & 0.03 & 0.04 & 0.05 & 0.00 & 0.04 & 0.45 & 0.01 & 0.03 & 0.04 & 0.05 \\
-0.5 & 0.00 & 0.00 & 0.00 & 0.00 & 0.00 & 0.00 & 0.00 & 0.00 & 0.00 & 0.00 & 0.00 & 0.00 & 0.00 & 0.00 \\
0 & 0.01 & 0.00 & 0.00 & 0.00 & 0.00 & 0.00 & 0.00 & 0.01 & 0.00 & 0.00 & 0.00 & 0.00 & 0.00 & 0.00 \\
0.5 & 0.05 & 0.00 & 0.00 & 0.00 & 0.00 & 0.00 & 0.00 & 0.05 & 0.00 & 0.01 & 0.00 & 0.00 & 0.00 & 0.00 \\
0.9 & 0.32 & 0.07 & 0.02 & 0.00 & 0.00 & 0.00 & 0.00 & 0.31 & 0.09 & 0.45 & 0.04 & 0.00 & 0.00 & 0.00 \\
0.95 & 0.41 & 0.10 & 0.04 & 0.01 & 0.00 & 0.00 & 0.00 & 0.40 & 0.10 & 0.65 & 0.05 & 0.00 & 0.00 & 0.00 \\
0.99 & 0.51 & 0.14 & 0.05 & 0.01 & 0.00 & 0.00 & 0.00 & 0.49 & 0.14 & 0.86 & 0.06 & 0.00 & 0.00 & 0.00 \\
& Forecasting Horizon: Four Quarters Ahead (h=4) & & & & & & & \\
$d \backslash \rho$ & -0.9 & -0.5 & 0 & 0.5 & 0.9 & 0.95 & 0.99 & -0.9 & -0.5 & 0 & 0.5 & 0.9 & 0.95 & 0.99 \\
\hline-0.9 & 0.00 & 0.00 & 0.01 & 0.01 & 0.04 & 0.04 & 0.05 & 0.00 & 0.04 & 0.45 & 0.01 & 0.04 & 0.04 & 0.05 \\
-0.5 & 0.00 & 0.00 & 0.00 & 0.00 & 0.00 & 0.00 & 0.00 & 0.00 & 0.00 & 0.01 & 0.00 & 0.00 & 0.00 & 0.00 \\
0 & 0.08 & 0.00 & 0.00 & 0.00 & 0.00 & 0.00 & 0.00 & 0.01 & 0.00 & 0.00 & 0.00 & 0.00 & 0.00 & 0.00 \\
0.5 & 0.05 & 0.00 & 0.00 & 0.00 & 0.00 & 0.00 & 0.00 & 0.05 & 0.00 & 0.01 & 0.00 & 0.00 & 0.00 & 0.00 \\
0.9 & 0.32 & 0.07 & 0.02 & 0.00 & 0.00 & 0.00 & 0.00 & 0.32 & 0.07 & 0.45 & 0.04 & 0.00 & 0.00 & 0.00 \\
0.95 & 0.42 & 0.10 & 0.04 & 0.01 & 0.00 & 0.00 & 0.00 & 0.40 & 0.10 & 0.65 & 0.05 & 0.00 & 0.00 & 0.00 \\
0.99 & 0.52 & 0.14 & 0.05 & 0.01 & 0.00 & 0.00 & 0.00 & 0.50 & 0.14 & 0.86 & 0.06 & 0.00 & 0.00 & 0.00 \\
\hline
\end{tabular}

Table continued on next page ... 
Table 1 continued

Panel B: $\mathbb{L}^{2}$ distance for minimized prediction error differences

Regular MIDAS Multiplicative MIDAS

Forecasting Horizon: Two Quarters Ahead $(\mathrm{h}=2)$

\begin{tabular}{rrrrrrrrrrrrrrrr}
$d \backslash \rho$ & -0.9 & -0.5 & 0 & 0.5 & 0.9 & 0.95 & 0.99 & -0.9 & -0.5 & 0 & 0.5 & 0.9 & 0.95 & 0.99 \\
\hline-0.9 & 0.90 & 0.86 & 0.89 & 0.91 & 0.98 & 1.01 & 1.05 & 0.31 & 1.04 & 0.45 & 0.51 & 1.01 & 0.92 & 0.83 \\
-0.5 & 0.02 & 0.04 & 0.02 & 0.06 & 0.08 & 0.08 & 0.09 & 0.02 & 0.02 & 0.01 & 0.01 & 0.02 & 0.03 & 0.02 \\
0 & 0.02 & 0.00 & 0.00 & 0.00 & 0.00 & 0.00 & 0.00 & 0.02 & 0.00 & 0.00 & 0.00 & 0.00 & 0.00 & 0.00 \\
0.5 & 0.09 & 0.00 & 0.01 & 0.00 & 0.01 & 0.03 & 0.10 & 0.10 & 0.01 & 0.01 & 0.02 & 0.01 & 0.01 & 0.01 \\
0.9 & 0.64 & 0.42 & 0.40 & 0.31 & 0.44 & 0.49 & 0.53 & 0.98 & 0.74 & 0.45 & 0.42 & 0.27 & 0.36 & 0.28 \\
0.95 & 0.58 & 0.74 & 0.71 & 0.56 & 0.61 & 0.64 & 0.67 & 1.35 & 1.05 & 0.65 & 0.64 & 0.40 & 0.39 & 0.42 \\
0.99 & 0.81 & 1.12 & 1.06 & 0.86 & 0.80 & 0.81 & 0.83 & 1.74 & 1.05 & 0.86 & 0.88 & 0.60 & 0.60 & 0.53
\end{tabular}

Forecasting Horizon: Four Quarters Ahead $(\mathrm{h}=4)$

\begin{tabular}{rcccccccccccccc}
$d \backslash \rho$ & -0.9 & -0.5 & 0 & 0.5 & 0.9 & 0.95 & 0.99 & -0.9 & -0.5 & 0 & 0.5 & 0.9 & 0.95 & 0.99 \\
\hline-0.9 & 0.91 & 0.87 & 0.88 & 0.92 & 0.98 & 1.01 & 1.06 & 0.28 & 1.05 & 0.45 & 0.52 & 0.87 & 0.86 & 0.67 \\
-0.5 & 0.02 & 0.04 & 0.02 & 0.06 & 0.08 & 0.08 & 0.09 & 0.02 & 0.02 & 0.01 & 0.01 & 0.02 & 0.01 & 0.03 \\
0 & 0.02 & 0.00 & 0.00 & 0.00 & 0.00 & 0.00 & 0.00 & 0.02 & 0.00 & 0.00 & 0.00 & 0.00 & 0.00 & 0.00 \\
0.5 & 0.08 & 0.00 & 0.01 & 0.00 & 0.01 & 0.02 & 0.05 & 0.09 & 0.01 & 0.01 & 0.02 & 0.01 & 0.01 & 0.01 \\
0.9 & 0.66 & 0.42 & 0.41 & 0.31 & 0.44 & 0.49 & 0.52 & 0.98 & 0.74 & 0.45 & 0.43 & 0.35 & 0.30 & 0.28 \\
0.95 & 0.91 & 0.74 & 0.71 & 0.56 & 0.61 & 0.64 & 0.66 & 1.36 & 1.06 & 0.65 & 0.65 & 0.40 & 0.52 & 0.40 \\
0.99 & 0.82 & 1.12 & 1.07 & 0.87 & 0.80 & 0.81 & 0.81 & 1.78 & 1.05 & 0.86 & 0.89 & 0.60 & 0.58 & 0.56 \\
\hline
\end{tabular}




\section{Table 2: Approximation Results between MIDAS and Two-Factor State Space Model}

Entries in Panel A pertain to the values of the $L^{2}$ measure appearing in equation (3.8) comparing Kalman Filter for the two-factor model appearing in section 3.2. In Panel B the entries pertain to the values of the $L^{2}$ when the MIDAS weights are optimized in terms of minimal prediction error - as appearing in equation (3.6). Entries cover both regular and multiplicative MIDAS regressions, with $d=d_{1}=d_{2}, \rho=\rho_{1}$ $=\rho_{2}, \gamma_{1,1}=\gamma_{1,2}=\gamma_{2,1}=\gamma_{2,2}=0.5$ and $\sigma_{\eta, 1}^{2}=\sigma_{\eta, 2}^{2}=\sigma_{y}^{2}=\sigma_{x}^{2}=1$. The multiplicative MIDAS scheme refers to the ADL-MIDAS regression appearing in equation (2.23), whereas the regular one refers to equation (3.5).

Panel A: $\mathbb{L}^{2}$ distance

\begin{tabular}{|c|c|c|c|c|c|c|c|c|c|c|c|c|c|c|}
\hline & \multicolumn{7}{|c|}{ Regular MIDAS } & \multicolumn{7}{|c|}{ Multiplicative MIDAS } \\
\hline & For & 19 & & & Quart & $\mathrm{Al}$ & We (II & & & & & & & \\
\hline$d \backslash \rho$ & -0.9 & -0.5 & 0 & 0.5 & 0.9 & 0.95 & 0.99 & -0.9 & -0.5 & 0 & 0.5 & 0.9 & 0.95 & 0.99 \\
\hline-0.9 & .00 & 0.00 & 0.00 & 0.00 & 0.00 & 0.01 & 0.0 & 0.00 & 0.00 & 0.00 & 0.00 & 0.00 & 0.01 & 0.00 \\
\hline-0. & 0.00 & 0.00 & 0.00 & 0.00 & 0.00 & 0.00 & 0. & & 0.00 & 0.00 & 0. & 0.00 & 0.00 & 0.00 \\
\hline 0 & 0.02 & 0.00 & 0.00 & 0.00 & 0.00 & 0.00 & 0. & 0.02 & 0.00 & 0.00 & 0.00 & 0.00 & 0.00 & 0.00 \\
\hline 0.5 & 0.04 & 0.00 & 0.00 & 0.00 & 0.00 & 0.00 & 0. & 0.04 & 0.00 & 0.00 & 0.00 & 0.00 & 0.00 & 0.00 \\
\hline 0.9 & 0.0 & 0.0 & 0.00 & 0.0 & 0.00 & 0.00 & 0 . & & 0.00 & 0.00 & 0.00 & 0.00 & 0.00 & 0.00 \\
\hline 0.95 & 0.0 & 0.0 & 0.00 & 0.0 & 0.00 & 0. & 0. & & 0.00 & 0.00 & 0.00 & 0.00 & 0.00 & 0.00 \\
\hline 0.99 & 0.09 & 0.00 & 0.00 & 0.00 & 0.00 & 0.00 & 0.00 & 0.10 & 0.00 & 0.00 & 0.00 & 0.00 & 0.00 & 0.00 \\
\hline
\end{tabular}

Forecasting Horizon: Four Quarters Ahead $(\mathrm{h}=4)$

\begin{tabular}{rcccccccccccccc}
\hline$d \backslash \rho$ & -0.9 & -0.5 & 0 & 0.5 & 0.9 & 0.95 & 0.99 & -0.9 & -0.5 & 0 & 0.5 & 0.9 & 0.95 & 0.99 \\
\hline-0.9 & 0.00 & 0.00 & 0.00 & 0.00 & 0.00 & 0.01 & 0.01 & 0.00 & 0.00 & 0.00 & 0.00 & 0.00 & 0.00 & 0.01 \\
-0.5 & 0.01 & 0.00 & 0.00 & 0.00 & 0.00 & 0.00 & 0.00 & 0.01 & 0.00 & 0.00 & 0.00 & 0.00 & 0.00 & 0.00 \\
0 & 0.02 & 0.00 & 0.00 & 0.00 & 0.00 & 0.00 & 0.00 & 0.02 & 0.00 & 0.00 & 0.00 & 0.00 & 0.00 & 0.00 \\
0.5 & 0.04 & 0.00 & 0.00 & 0.00 & 0.00 & 0.00 & 0.00 & 0.04 & 0.00 & 0.00 & 0.00 & 0.00 & 0.00 & 0.00 \\
0.9 & 0.08 & 0.00 & 0.00 & 0.00 & 0.00 & 0.00 & 0.00 & 0.09 & 0.00 & 0.00 & 0.00 & 0.00 & 0.00 & 0.00 \\
0.95 & 0.09 & 0.00 & 0.00 & 0.00 & 0.00 & 0.00 & 0.00 & 0.09 & 0.00 & 0.00 & 0.00 & 0.00 & 0.00 & 0.00 \\
0.99 & 0.09 & 0.00 & 0.00 & 0.00 & 0.00 & 0.00 & 0.00 & 0.10 & 0.00 & 0.00 & 0.00 & 0.00 & 0.00 & 0.00 \\
\hline
\end{tabular}

Table continued on next page ... 
Table 2 continued

Panel B: $\mathbb{L}^{2}$ distance for minimized prediction error differences

Regular MIDAS Multiplicative MIDAS

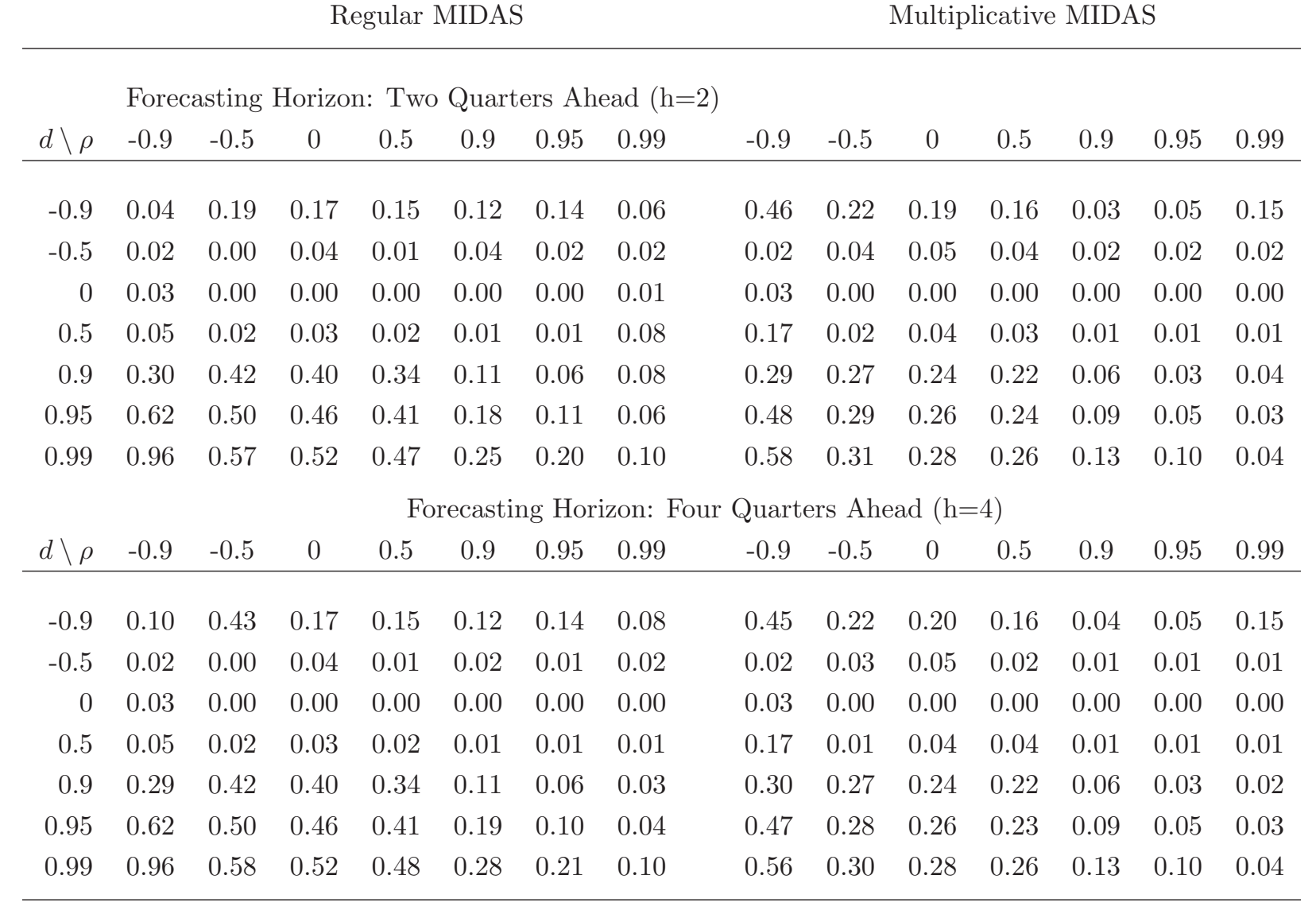


Table 3: Ratio of Prediction Errors: MIDAS/One-Factor State Space Model $\rho_{1}=\rho_{2}=\rho, d_{1}=d_{2}=d$

Entries pertain to the the minimized values of the prediction error objective function appearing in equation (3.6). The ratio is computed for a one-factor Kalman Filter (denoted SS1) and regular MIDAS (see equation (3.5)). The data generating process is a two-factor model appearing in section 3.2. Entries pertain to three prediction horizons $h=2$ and 4 .

\begin{tabular}{|c|c|c|c|c|c|c|c|c|c|c|c|c|c|c|}
\hline \multicolumn{8}{|c|}{ Panel A Regular MIDAS } & \multicolumn{7}{|c|}{ Panel B Multiplicative MIDAS } \\
\hline \multicolumn{15}{|c|}{ Forecasting Horizon: Two Quarters Ahead $(\mathrm{h}=2)$} \\
\hline \multicolumn{8}{|c|}{ PE-MIDAS / PE-SS1 } & \multicolumn{7}{|c|}{ PE-MIDAS / PE-SS1 } \\
\hline$d \backslash \rho$ & -0.9 & -0.5 & 0 & 0.5 & 0.9 & 0.95 & 0.99 & -0.9 & -0.5 & 0 & 0.5 & 0.9 & 0.95 & 0.99 \\
\hline-0.9 & 0.92 & 0.66 & 0.64 & 0.66 & 0.73 & 0.81 & 0.94 & 0.92 & 0.66 & 0.64 & 0.70 & 0.73 & 0.81 & 0.94 \\
\hline-0.5 & 0.91 & 0.75 & 0.72 & 0.75 & 0.88 & 0.93 & 0.98 & 0.91 & 0.75 & 0.72 & 0.75 & 0.88 & 0.93 & 0.98 \\
\hline 0 & 0.92 & 0.79 & 0.75 & 0.79 & 0.92 & 0.96 & 0.99 & 0.92 & 0.79 & 0.75 & 0.79 & 0.92 & 0.96 & 0.99 \\
\hline 0.5 & 0.88 & 0.75 & 0.72 & 0.75 & 0.91 & 0.96 & 1.00 & 0.88 & 0.75 & 0.72 & 0.75 & 0.91 & 0.96 & 1.00 \\
\hline 0.9 & 0.73 & 0.63 & 0.62 & 0.66 & 0.92 & 1.03 & 1.17 & 0.73 & 0.63 & 0.62 & 0.66 & 0.92 & 1.03 & 1.17 \\
\hline 0.95 & 0.69 & 0.60 & 0.60 & 0.65 & 0.90 & 1.06 & 1.25 & 0.69 & 0.60 & 0.61 & 0.65 & 0.90 & 1.06 & 1.25 \\
\hline 0.99 & 0.67 & 0.58 & 0.59 & 0.64 & 0.82 & 0.98 & 1.35 & 0.67 & 0.58 & 0.59 & 0.64 & 0.82 & 0.98 & 1.35 \\
\hline \multicolumn{15}{|c|}{ Forecasting Horizon: Four Quarters Ahead $(\mathrm{h}=4)$} \\
\hline \multicolumn{8}{|c|}{ PE-MIDAS / PE-SS1 } & \multicolumn{7}{|c|}{ PE-MIDAS / PE-SS1 } \\
\hline$d \backslash \rho$ & -0.9 & -0.5 & 0 & 0.5 & 0.9 & 0.95 & 0.99 & -0.9 & -0.5 & 0 & 0.5 & 0.9 & 0.95 & 0.99 \\
\hline-0.9 & 1.09 & 0.66 & 0.64 & 0.66 & 0.72 & 0.80 & 0.94 & 0.92 & 0.66 & 0.64 & 0.72 & 0.72 & 0.80 & 0.94 \\
\hline-0.5 & 0.91 & 0.75 & 0.72 & 0.75 & 0.89 & 0.93 & 0.98 & 0.91 & 0.75 & 0.72 & 0.75 & 0.89 & 0.93 & 0.98 \\
\hline 0 & 0.92 & 0.79 & 0.75 & 0.79 & 0.92 & 0.96 & 0.99 & 0.92 & 0.79 & 0.75 & 0.79 & 0.92 & 0.96 & 0.99 \\
\hline 0.5 & 0.89 & 0.75 & 0.72 & 0.75 & 0.91 & 0.95 & 0.99 & 0.89 & 0.75 & 0.72 & 0.75 & 0.91 & 0.95 & 0.99 \\
\hline 0.9 & 0.72 & 0.63 & 0.62 & 0.66 & 0.92 & 1.01 & 1.10 & 0.72 & 0.63 & 0.62 & 0.66 & 0.92 & 1.01 & 1.10 \\
\hline 0.95 & 0.69 & 0.61 & 0.61 & 0.64 & 0.92 & 1.06 & 1.19 & 0.69 & 0.61 & 0.61 & 0.64 & 0.92 & 1.06 & 1.19 \\
\hline 0.99 & 0.67 & 0.59 & 0.59 & 0.63 & 0.87 & 1.04 & 1.35 & 0.67 & 0.59 & 0.59 & 0.63 & 0.87 & 1.04 & 1.35 \\
\hline
\end{tabular}


Table 4: The Ratio of Prediction Error: MIDAS / One-Factor State Space Model $\rho_{1} \neq \rho_{2}, d_{1}=d_{2}=0$

Entries pertain to the minimized values of the prediction error objective function appearing in equation (3.6). The ratio is computed for a one-factor Kalman Filter (denoted SS1) and regular MIDAS (see equation (3.5)). The data generating process is a two-factor model appearing in section 3.2. Entries pertain to three prediction horizons $h=2$ and 4.

Panel A Regular MIDAS

Panel B Multiplicative MIDAS

\begin{tabular}{|c|c|c|c|c|c|c|c|c|c|c|c|c|c|c|}
\hline & \multicolumn{7}{|c|}{ Panel A Regular MIDAS } & \multicolumn{7}{|c|}{ Panel B Multiplicative MIDAS } \\
\hline \multicolumn{15}{|c|}{ Forecasting Horizon: Two Quarters Ahead $(\mathrm{h}=2)$} \\
\hline \multicolumn{8}{|c|}{ PE-MIDAS / PE-SS1 } & \multicolumn{7}{|c|}{ PE-MIDAS / PE-SS1 } \\
\hline$\rho_{2} \backslash \rho_{1}$ & -0.9 & -0.5 & 0 & 0.5 & 0.9 & 0.95 & 0.99 & -0.9 & -0.5 & 0 & 0.5 & 0.9 & 0.95 & 0.99 \\
\hline-0.9 & 0.65 & 0.57 & 0.56 & 0.59 & 0.78 & 0.77 & 0.89 & 1.02 & 0.95 & 0.94 & 0.98 & 1.00 & 1.00 & 1.00 \\
\hline-0.5 & 0.80 & 0.61 & 0.58 & 0.61 & 0.78 & 0.84 & 0.93 & 1.02 & 0.99 & 0.96 & 0.99 & 1.11 & 1.16 & 1.28 \\
\hline 0 & 0.81 & 0.61 & 0.58 & 0.60 & 0.76 & 0.82 & 0.92 & 1.21 & 0.94 & 0.90 & 0.94 & 1.17 & 1.24 & 1.39 \\
\hline 0.5 & 0.80 & 0.59 & 0.56 & 0.59 & 0.75 & 0.81 & 0.95 & 1.18 & 0.91 & 0.87 & 0.91 & 1.11 & 1.18 & 1.36 \\
\hline 0.9 & 0.93 & 0.67 & 0.64 & 0.67 & 0.75 & 0.79 & 0.87 & 1.00 & 1.00 & 0.98 & 1.00 & 0.98 & 0.98 & 1.05 \\
\hline 0.95 & 0.87 & 0.68 & 0.66 & 0.68 & 0.74 & 0.77 & 0.81 & 1.00 & 0.95 & 0.91 & 0.94 & 1.01 & 1.00 & 1.02 \\
\hline 0.99 & 0.89 & 0.69 & 0.67 & 0.72 & 0.74 & 0.74 & 0.74 & 1.08 & 1.12 & 1.11 & 1.17 & 1.08 & 1.02 & 1.00 \\
\hline \multicolumn{15}{|c|}{ Forecasting Horizon: Four Quarters Ahead $(\mathrm{h}=4)$} \\
\hline \multicolumn{8}{|c|}{ PE-MIDAS / PE-SS1 } & \multicolumn{7}{|c|}{ PE-MIDAS / PE-SS1 } \\
\hline$\rho_{2} \backslash \rho_{1}$ & -0.9 & -0.5 & 0 & 0.5 & 0.9 & 0.95 & 0.99 & -0.9 & -0.5 & 0 & 0.5 & 0.9 & 0.95 & 0.99 \\
\hline-0.9 & 0.78 & 0.69 & 0.68 & 0.72 & 0.97 & 0.94 & 1.00 & 1.01 & 0.95 & 0.93 & 0.96 & 1.03 & 1.05 & 1.08 \\
\hline-0.5 & 0.90 & 0.69 & 0.65 & 0.69 & 0.88 & 0.94 & 1.00 & 1.13 & 0.95 & 0.91 & 0.95 & 1.11 & 1.16 & 1.21 \\
\hline 0 & 0.91 & 0.68 & 0.65 & 0.68 & 0.87 & 0.93 & 0.98 & 1.20 & 0.90 & 0.86 & 0.90 & 1.15 & 1.22 & 1.29 \\
\hline 0.5 & 0.94 & 0.69 & 0.66 & 0.69 & 0.88 & 0.94 & 1.01 & 1.17 & 0.93 & 0.89 & 0.92 & 1.11 & 1.17 & 1.23 \\
\hline 0.9 & 1.13 & 0.94 & 0.90 & 0.92 & 0.99 & 1.01 & 1.05 & 1.01 & 1.04 & 1.03 & 1.05 & 0.98 & 1.01 & 1.05 \\
\hline 0.95 & 1.04 & 0.96 & 0.93 & 0.95 & 1.01 & 1.00 & 1.02 & 1.04 & 0.95 & 0.92 & 0.95 & 1.01 & 1.00 & 1.02 \\
\hline 0.99 & 1.06 & 0.97 & 0.94 & 0.97 & 1.05 & 1.02 & 0.99 & 1.06 & 0.99 & 0.96 & 0.99 & 1.05 & 1.02 & 1.00 \\
\hline
\end{tabular}


Table 5: Definition of the Regressors

\begin{tabular}{llcc}
\hline Name & Description & Period & Transformation \\
\hline TERM & Term spread (10yr T-Bond - 1yr T-bond) & $1959: 01-2009: 05$ & lv \\
SP500 & Stock Price Index: Standard \& Poor's 500 Composite & $1959: 01-2009: 05$ & $\Delta \ln$ \\
IP & Industry Production Index (SA) & $1959: 01-2009: 05$ & $\Delta \ln$ \\
Emply & All Employees: Total Nonfarm Payroll(SA, Thous) & $1959: 01-2009: 05$ & $\Delta \ln$ \\
Exptn & Consumer Expectations (Q1-66=100) & $1959: 01-2009: 05$ & $\ln$ \\
PI & Personal Income less Transfer Payments (SAAR) & $1959: 02-2009: 05$ & $\Delta \ln$ \\
LEI & Leading index, percent change from previous month & $1959: 02-2009: 05$ & $\operatorname{lv}$ \\
Manu & Real Manufacturing \& Trade Inventories: All Industries (SA) & $1967: 02-2009: 05$ & $\Delta \ln$ \\
Oil & Crude Oil Spot Price: WTI Cushing & $1982: 01-2009: 05$ & $\Delta \ln$ \\
\hline
\end{tabular}

Data Source: Federal Reserve Board and Haver Analytics. 


\section{Table 6: RMSE Forecasting Comparison for MIDAS and State Space Model}

We use a dataset with mixed frequencies: monthly and quarterly. The forecasting variable is the growth rate of real GDP from 1959Q1 to 2009Q1. Each model consists of real GDP growth and one of the monthly indicators, with the latter as observed up to the second month of each quarter. Table 5 contains the details on the definitions and data transformations. We use the root mean squared forecasting error (RMSE) to evaluate each models forecasting accuracy. Section 4 contains the sample configurations.

\begin{tabular}{lcccccccc} 
h (Quarters) & 1 & 2 & 3 & 4 & 5 & 6 & 7 & 8 \\
Term Spread & & & & & & & & \\
\hline State Space (m0) & 1.06 & 0.97 & 1.06 & 1.02 & 0.99 & 1.00 & 0.97 & 1.00 \\
Regular Midas (m1) & 0.82 & 0.81 & 0.85 & 0.80 & 0.85 & 0.78 & 0.81 & 0.83 \\
Multiple Midas (m2) & 0.80 & 0.84 & 0.84 & 0.81 & 0.85 & 0.78 & 0.87 & 0.86 \\
Ratio (m0/m1) & 1.29 & 1.19 & 1.25 & 1.28 & 1.16 & 1.29 & 1.20 & 1.21 \\
Ratio (m0/m2) & 1.33 & 1.16 & 1.26 & 1.26 & 1.16 & 1.28 & 1.12 & 1.17
\end{tabular}

\begin{tabular}{lllllllll} 
S\&P 500 & \multicolumn{1}{c}{0.77} & 0.77 & 0.77 & 0.78 & 0.74 & 0.77 & 0.80 \\
State Space (m0) & 0.81 & 0.77 & 0.82 & 0.84 \\
Regular Midas (m1) & 0.79 & 0.82 & 0.82 & 0.84 & 0.84 & 0.82 & 0.84 & 0.84 \\
Multiple Midas (m2) & 0.80 & 0.87 & 0.81 & 0.85 & 0.85 & 0.81 & 0.82 & 0.82 \\
Ratio (m0/m1) & 1.02 & 0.94 & 0.94 & 0.92 & 0.93 & 0.90 & 0.91 & 0.95 \\
Ratio (m0/m2) & 1.01 & 0.88 & 0.96 & 0.91 & 0.92 & 0.91 & 0.93 & 0.97 \\
& & & & & & & & \\
Industrial Production & & & & & & & & \\
\hline State Space (m0) & 0.75 & 0.76 & 0.81 & 0.81 & 0.80 & 0.75 & 0.77 & 0.81 \\
Regular Midas (m1) & 0.78 & 0.86 & 0.83 & 0.86 & 0.84 & 0.79 & 0.81 & 0.81 \\
Multiple Midas (m2) & 0.79 & 0.86 & 0.85 & 0.87 & 0.84 & 0.80 & 0.82 & 0.79 \\
Ratio (m0/m1) & 0.96 & 0.88 & 0.98 & 0.94 & 0.95 & 0.95 & 0.95 & 1.00 \\
Ratio (m0/m2) & 0.96 & 0.88 & 0.96 & 0.93 & 0.95 & 0.94 & 0.93 & 1.03
\end{tabular}

Table continued on next page ... 
Table 6 (Cont'd) RMSE Forecasting Comparison for MIDAS and State Space Model

$\begin{array}{lcccccccc}\text { h (Quarters) } & 1 & 2 & 3 & 4 & 5 & 6 & 7 & 8 \\ \text { Employment } & & & & & & & & \\ \text { State Space (m0) } & 0.72 & 0.81 & 0.84 & 0.85 & 0.88 & 0.79 & 0.81 & 0.84 \\ \text { Regular Midas (m1) } & 0.78 & 0.83 & 0.82 & 0.84 & 0.81 & 0.80 & 0.80 & 0.79 \\ \text { Multiple Midas (m2) } & 0.78 & 0.84 & 0.83 & 0.85 & 0.82 & 0.81 & 0.80 & 0.79 \\ \text { Ratio (m0/m1) } & 0.93 & 0.97 & 1.02 & 1.01 & 1.09 & 1.00 & 1.01 & 1.07 \\ \text { Ratio }(\mathrm{m} 0 / \mathrm{m} 2) & 0.93 & 0.96 & 1.01 & 1.00 & 1.07 & 0.98 & 1.01 & 1.08\end{array}$

Consumer Expectations

$\begin{array}{lllllllll}\text { State Space }(\mathrm{m} 0) & 0.96 & 1.02 & 1.07 & 1.06 & 1.09 & 1.07 & 1.07 & 1.07 \\ \text { Regular Midas }(\mathrm{m} 1) & 0.74 & 0.82 & 0.79 & 0.84 & 0.84 & 0.81 & 0.83 & 0.83 \\ \text { Multiple Midas }(\mathrm{m} 2) & 0.74 & 0.82 & 0.80 & 0.84 & 0.84 & 0.79 & 0.83 & 0.83 \\ \text { Ratio }(\mathrm{m} 0 / \mathrm{m} 1) & 1.30 & 1.24 & 1.34 & 1.26 & 1.31 & 1.33 & 1.28 & 1.30 \\ \text { Ratio }(\mathrm{m} 0 / \mathrm{m} 2) & 1.30 & 1.24 & 1.34 & 1.26 & 1.31 & 1.34 & 1.29 & 1.30\end{array}$

Personal Income

$\begin{array}{lllllllll}\text { State Space }(\mathrm{m} 0) & 0.73 & 0.78 & 0.81 & 0.79 & 0.76 & 0.74 & 0.77 & 0.80 \\ \text { Regular Midas }(\mathrm{m} 1) & 0.87 & 0.98 & 0.84 & 0.87 & 0.82 & 0.84 & 0.84 & 0.81 \\ \text { Multiple Midas }(\mathrm{m} 2) & 0.94 & 1.00 & 0.92 & 0.91 & 0.82 & 0.85 & 0.89 & 0.84 \\ \text { Ratio }(\mathrm{m} 0 / \mathrm{m} 1) & 0.84 & 0.79 & 0.96 & 0.91 & 0.93 & 0.89 & 0.93 & 0.99 \\ \text { Ratio }(\mathrm{m} 0 / \mathrm{m} 2) & 0.78 & 0.77 & 0.87 & 0.87 & 0.93 & 0.87 & 0.87 & 0.95\end{array}$


Table 6 (Cont'd) RMSE Forecasting Comparison for MIDAS and State Space Model

\begin{tabular}{lcccccccc} 
h (Quarters) & 1 & 2 & 3 & 4 & 5 & 6 & 7 & 8 \\
Leading Index (LEI) & & & & & & & & \\
\hline State Space (m0) & 0.74 & 0.72 & 0.78 & 0.79 & 0.74 & 0.74 & 0.74 & 0.77 \\
Regular Midas (m1) & 0.66 & 0.81 & 0.81 & 0.79 & 0.76 & 0.78 & 0.83 & 0.84 \\
Multiple Midas (m2) & 0.68 & 0.82 & 0.79 & 0.78 & 0.77 & 0.80 & 0.84 & 0.81 \\
Ratio (m0/m1) & 1.11 & 0.89 & 0.96 & 1.00 & 0.96 & 0.94 & 0.90 & 0.92 \\
Ratio $(\mathrm{m} 0 / \mathrm{m} 2)$ & 1.09 & 0.88 & 1.00 & 1.01 & 0.95 & 0.93 & 0.88 & 0.95
\end{tabular}

Manufacturing

\begin{tabular}{lcccccccc} 
State Space (m0) & 0.90 & 0.88 & 0.88 & 0.86 & 0.80 & 0.81 & 0.84 & 0.87 \\
Regular Midas (m1) & 1.03 & 1.02 & 0.96 & 0.89 & 0.86 & 0.87 & 0.94 & 0.93 \\
Multiple Midas (m2) & 1.08 & 1.02 & 0.97 & 0.94 & 0.84 & 0.88 & 0.96 & 0.97 \\
Ratio (m0/m1) & 0.87 & 0.87 & 0.91 & 0.97 & 0.92 & 0.92 & 0.89 & 0.94 \\
Ratio (m0/m2) & 0.83 & 0.87 & 0.90 & 0.92 & 0.96 & 0.91 & 0.87 & 0.90 \\
& & & & & & & & \\
Crude Oil Price & & & & & & & & \\
\hline & & & & & & & & \\
State Space (m0) & 0.69 & 0.65 & 0.68 & 0.67 & 0.70 & 0.70 & 0.80 & 0.89 \\
Regular Midas (m1) & 0.65 & 0.76 & 0.70 & 0.74 & 0.72 & 0.80 & 0.90 & 0.95 \\
Multiple Midas (m2) & 0.65 & 0.77 & 0.72 & 0.76 & 0.70 & 0.81 & 0.92 & 0.97 \\
Ratio (m0/m1) & 1.06 & 0.86 & 0.97 & 0.91 & 0.97 & 0.87 & 0.89 & 0.94 \\
Ratio (m0/m2) & 1.06 & 0.85 & 0.95 & 0.88 & 0.99 & 0.86 & 0.88 & 0.92
\end{tabular}

